\title{
Performance measures for classification systems with rejection
}

\author{
Filipe Condessa ${ }^{\mathrm{a}}$, José Bioucas-Dias ${ }^{\mathrm{b}}$, Jelena Kovačevića ${ }^{\mathrm{a}}$ \\ ${ }^{a}$ Carnegie Mellon University, USA \\ ${ }^{b}$ Instituto de Telecomunicações, Instituto Superior Técnico, Universidade de Lisboa, \\ Portugal
}

\begin{abstract}
Classifiers with rejection are essential in real-world applications where misclassifications and their effects are critical. However, if no problem specific cost function is defined, there are no established measures to assess the performance of such classifiers. We introduce a set of desired properties for performance measures for classifiers with rejection, based on which we propose a set of three performance measures for the evaluation of the performance of classifiers with rejection. The nonrejected accuracy measures the ability of the classifier to accurately classify nonrejected samples; the classification quality measures the correct decision making of the classifier with rejector; and the rejection quality measures the ability to concentrate all misclassified samples onto the set of rejected samples. We derive the concept of relative optimality that allows us to connect the measures to a family of cost functions that take into account the trade-off between rejection and misclassification. We illustrate the use of the proposed performance measures on classifiers with rejection applied to synthetic and real-world data.

Keywords: classification with rejection, performance measures
\end{abstract}




\section{Introduction}

Classification with rejection is a viable option in real world applications of machine learning and pattern recognition, where the presence and cost of errors can be detrimental to the performance of automated classification systems.

Classification with rejection is often used in situations where the cost and consequences of misclassification are high, such as in automated medical diagnosis $[1,2,3,4,5]$, landcover classification in remote sensing $[6,7,8]$, automated software detection prediction [9], or large-scale OCR applications [10]. Another interesting situation for the use of classification with rejection is the design of classification systems through a cascading architecture, used in text categorization systems $[11,12]$, in time-series classification [13], or in medical diagnosis [14]. Another application for classification with rejection is associated to cases where samples might be of no interest to the application, as in image retrieval [15], object classification [16], or in scene identification [17]. A classifier with rejection can also cope with unknown information, reducing the threat posed by the existence of unknown samples or mislabeled training samples that hamper the performance pf the classifier.

Frequently, rejected samples are divided into two distinct classes: confusion or ambiguity rejection and distance rejection [18, 19, 20, 21]. This division is also present in [22], where the distinction is made between native (belonging to the known classes) and foreign (belonging to unknown classes) elements. The fundamental idea behind this division is the distinction between inlier samples that are equally likely to belong to more than one class (confusion or ambiguity reject, or native samples), and outlier samples that 
are not likely to belong to any class (distance reject or foreign samples).

This distinction between confusion reject and distance reject is useful when the decision to reclassify the rejected samples exist. This is central to the concept of fuzzy classification with rejection $[23,24,25,26,27,28]$. In [29], the distinction between confusion reject and distance reject is also associated to the concept of multivalued classifiers with rejection, where, instead of a classification (or rejection) being assigned to a label, a subset of classes is assigned to the label while the remaining classes are rejected. However, as in this paper we focus on the evaluation of the performance of classification with rejection, without considering reclassification of rejected samples, we do not make such distinction.

Classification with rejection was first analyzed in [30], where Chow's rule for optimum error-reject trade-off was presented. In a binary classification setting, Chow's rule allows for the determination of a threshold for rejection such that the classification risk is minimized. This requires both the knowledge of the a posterior probabilities and the existence of a cost function that specifies the cost of misclassification and the cost of rejection.

Multiple other designs for incorporating rejection into classification exist. In a binary classification setting, the reject option can be embedded in the classifier. An embedded reject option is possible through a risk minimization approach with the use of a hinge function, such as in [31, 32, 33], to minimize classification risk. It can also be achieved with support vector machines with embedded reject options, as explored in [34, 35, 36, 37].

These embedded designs can also be extended to a rejection framework in multiclass classification setting [38]. In [39], rejection in multiclass classi- 
fication settings is achieved through the use of a Bayesian combination rule to multi-expert systems, whereas in [40] rejection in a multi-class setting is achieved through the combination of multiple single-class classifiers. In [41], the authors design surrogate functions for the risk of classification with rejection tailored for the multiclass classification problem. In practice, the use of soft margin classifiers allows an easy extension of the use of classifiers with rejection from a binary to a multiclass situation.

There is no adequate standard measure for the assessment of the performance of a classifier with rejection. Accuracy-rejection curves (ARC), used in $[6,34,42,43,44,45]$, albeit popular in practical applications of classification with rejection have significant drawbacks. The ARC can also be represented as error rate vs. rejection curves $[46,47]$.

In [44], the problem of comparing performance of classifiers with rejection is approached through the use of ARC. Two classifiers with rejection, where a sweep on all possible values of rejection rate (rate between rejected samples and total number of samples) is performed for each of the classifiers with rejection. The classifiers can then be compared through an analysis of the behavior of the respective ARC. Three different pairwise behaviors are defined: crossing-over where the ARC of one of the classifiers cuts the ARC of the other classifier, diverging where the ARC of the classifiers is close near no-rejection and all-rejection scenarios and diverges in the other situations, and evenly spaced where the distance between the ARCs is similar at different rejected rates.

The use of ARC and the three classes of pairwise behavior for performance analysis of classifiers with rejection allows the pairwise comparison of classi- 
fiers with rejection when a sweep on the rejection rate of each of the classifier is possible. It allows the comparison, given either fixed rejection rate or a fixed desired accuracy, between the performance of two classifiers. However, it is unable to identify for which rejection rate is the performance better, or to compare two different classifiers when working at different rejection rates and values of accuracy of the nonrejected samples.

Another measure used for the assessment is the $F_{1}$ score $[11,48,38,9]$, where, based on extensions of the concept of precision and recall measured on the subset of samples that are not rejected, the $F_{1}$ score is computed.

Furthermore, obtaining sufficient points for an ARC might not be feasible for a classifier with embedded reject option, which requires retraining the classifier to achieve a different rejection ratio, or for classifiers that combine contextual information with rejection, such as $[5,8]$. This means that $\mathrm{ARC}$ and their $F_{1}$ equivalent, in the real world, are not able to describe the behavior of the classifier with rejection in all cases.

In [29], the normalized area under the ARC is used as performance measure. This corresponds to, given an ARC, computing the ratio between the integral of the difference between the ARC curve and the initial accuracy (for no rejection), and the integral difference between a perfect accuracy (1) and the initial accuracy (for no rejection). This suffers from the same constraints associated with obtaining a sufficient number of points for an ARC, and provides no information about interesting points of operation of the classifier with rejection.

In [49], a different approach is taken. A 3D ROC (receiver operating characteristic) plot of a $2 \mathrm{D}$ ROC surface is obtained by decomposing the false 
positive rate into false positive rate for outliers belonging to known classes and false positive rate for outliers belonging to unknown classes, with the VUC (volume under the curve) as the performance measure. With effect, $\mathrm{ROC}$ can be used during the training procedure of classifiers with rejection [35] or for derivation of rejection rules $[50,51,52]$. In [53], the authors establish a connection between Chow's formulation for rejection [30] and the derivations of rejection rules from ROC curves. However, the use of ROC curves for the analysis of the performance suffers from the same problems associated with ARC.

To fill this gap, we propose a set of desired properties for performance measures for classifiers with rejection, and a set of three performance measures that satisfy those properties.

A performance measure that evaluates the performance of a rejection mechanism, a procedure that decides which samples should be rejected and which samples should not be rejected, given a classifier should satisfy the following: Property I - be a function of the fraction of rejected samples; Property II — be able to compare different rejection mechanisms working at the same fraction of rejected samples; Property III — be able to compare rejection mechanisms working at a different fractions of rejected samples when one rejection mechanism outperforms the other; Property IV — be maximum for a rejection mechanism that no other feasible rejection mechanism outperforms, and minimum for a rejection mechanism that all other feasible rejection mechanisms outperform.

These properties rely on being able to state whether one rejection mechanism qualitatively outperforms the other. If a cost function exists that takes 
in account the cost of rejection and misclassification, the concept of outperformance is trivial, and this cost function not only satisfies the properties but is also the ideal performance measure for the problem in hand. It might not be feasible, however, to design a cost function for each individual classification problem. Thus, we derive a set of cases where the concept of outperformance is independent from a specific cost function (under the assumption that the cost of rejection is never greater than the cost of misclassification).

With the properties and the concept of outperformance in place, we present three measures that satisfy the above properties: Nonrejected accuracy measures the ability of the classifier to accurately classify nonrejected samples; Classification quality measures the ability of the classifier with rejection to accurately classify nonrejected samples and to reject misclassified samples; Rejection quality measures the ability of the classifier with rejection to make errors on rejected samples only.

With the three measures in place, we can explore the best and worst case scenarios for each measure, for a given reference classifier with rejection. We denote the proximity of a classifier with rejection to its best and worst case scenarios, with regard to a reference classifier with rejection, as relative optimality. This allows us to easily connect performance measures to problem specific cost functions. For a classifier with rejection that rejects at two different numbers of rejected samples, the relative optimality defines the family of cost functions on which rejection at one number rejected samples is better, equal, or worse than rejection at the other number of rejected samples.

The main contribution of this paper is the expansion of the set of performance measures for evaluation of classification with rejection, currently 
centered only on the nonrejected accuracy as the core component of ARC. To this extent, the follow contributions are made. The development of a cost-function agnostic concept of outperformance for classifiers with rejection; the definition of general properties that performance should hold; the presentation of three performance measures; and the connection of the performance measures to families of cost-functions for which outperformance is clearly defined based on the concept of relative optimality.

The nonrejected accuracy corresponds to the classification accuracy on the subset of nonrejected samples, and has been extensively used as the core component of ARC for evaluation of the performance of classifiers with rejection. The concepts of classification quality, and the rejection quality as performance measures are novel.

The rest of the paper is structured as follows. In Section 2, we present the concept of classifier with rejection, introduce the necessary notation, define three concepts of rejector outperformance that do not depend on cost functions, and present the desired performance measure properties. In Section 3, we present the set of proposed performance measures. In Section 4, we connect the performance measures to cost functions by defining relative optimality. In Section 5, we illustrate performance measures on real-world applications. In Section 6, we conclude the paper.

\section{Classifiers with rejection}

\subsection{Preliminary definition}

We begin by defining the basic notation for classifiers with rejection. Without loss of generality, a classifier with rejection can be seen as a coupling 
of a classifier $C$ with a rejection system $R$. The classification $\hat{\boldsymbol{y}}=C(\boldsymbol{x})$ results from a mapping of $n d$-dimensional feature vectors $x$ into $n$ labels $C: \mathcal{R}^{d \times n} \rightarrow\{1, \ldots, K\}^{n}$. The rejection $\boldsymbol{r}=R(\boldsymbol{x}, \hat{\boldsymbol{y}})$ results from a mapping of the classification (feature vectors $\boldsymbol{x}$ and associated labels $\hat{\boldsymbol{y}}=C(\boldsymbol{x})$ ) into a binary rejection vector, $R: \mathcal{R}^{d \times n} \times\{1, \ldots, K\}^{n} \rightarrow\{0,1\}^{n}$. We thus define a classification with rejection $\hat{\boldsymbol{y}}^{R}$ as $\hat{\boldsymbol{y}}_{i}^{R}=\hat{\boldsymbol{y}}_{i}$ if $\boldsymbol{r}_{i}=0$, and $\hat{\boldsymbol{y}}_{i}^{R}=0$ if $\boldsymbol{r}_{i}=1$, where $\hat{\boldsymbol{y}}_{i}$ corresponds to the classification of the $i$ th sample, $\boldsymbol{r}_{i}$ corresponds to the binary decision to reject $\left(r_{i}=1\right)$ or not $\left(r_{i}=0\right)$ the $i$ th sample, and $\hat{\boldsymbol{y}}_{i}^{R}=0$ denotes rejection.

By comparing the classification $\hat{\boldsymbol{y}}$ with its ground truth $\boldsymbol{y}$, we form a binary $n$-dimensional accuracy vector $\boldsymbol{a}$, where $\boldsymbol{a}_{i}=1$ and $\boldsymbol{a}_{i}=0$ correspond to an accurately classified and a misclassified $i$ th sample, respectively.

\subsection{Notation}

The binary vector $\boldsymbol{a}$ imposes a partition of the set of samples in two subsets $\mathcal{A}$ and $\mathcal{M}$, namely the subset of accurately classified samples and the subset of misclassified samples. Let $\boldsymbol{c}$ be a confidence vector associated with the classification $\hat{y}$, such that $\boldsymbol{c}_{i} \geq \boldsymbol{c}_{j} \Longrightarrow \boldsymbol{r}_{i} \leq \boldsymbol{r}_{j}$, which implies that if sample $i$ is rejected, then all the samples $j$ with smaller confidence $\boldsymbol{c}_{i}<\boldsymbol{c}_{j}$ are also rejected. We have then the ground truth $\boldsymbol{y}$, the result of the classification $\hat{\boldsymbol{y}}$, and the result of the classification with rejection $\hat{\boldsymbol{y}}^{R}$.

Let $\boldsymbol{c} \downarrow$ denote the reordering of the confidence vector $\boldsymbol{c}$ in decreasing order. If we keep $k$ samples with the highest confidence and reject the rest $n-k$ samples, we obtain two subsets: $k$ nonrejected samples and $n-k$ 
rejected samples, $\mathcal{N}$ and $\mathcal{R}^{1}$ respectively. Our goal is to separate the accuracy vector $\boldsymbol{a}$ into two subvectors $\left(\boldsymbol{a}_{\mathcal{N}}\right.$ and $\left.\boldsymbol{a}_{\mathcal{R}}\right)$, based on the confidence vector $\boldsymbol{c}$ such that all misclassifications are in the $\boldsymbol{a}_{\mathcal{R}}$ subvector, and all accurate classifications are in the $\boldsymbol{a}_{\mathcal{N}}$ subvector. We should note that, since $\mathcal{N}$ and $\mathcal{R}$ have disjoint supports,

$$
\|\boldsymbol{a}\|_{0}=\left\|\boldsymbol{a}_{\mathcal{N}}\right\|_{0}+\left\|\boldsymbol{a}_{\mathcal{R}}\right\|_{0}
$$

for all $\mathcal{N}, \mathcal{R}$ such that $\mathcal{N} \cap \mathcal{R}=\emptyset$ and $\mathcal{N} \cup \mathcal{R}=\{1, \ldots, n\}$, meaning that the number of accurately classified samples $\|\boldsymbol{a}\|_{0}$ is equal to the sum of the number of accurately classified samples not rejected $\left\|\boldsymbol{a}_{\mathcal{N}}\right\|_{0}$ with the number of accurately classified samples rejected $\left\|\boldsymbol{a}_{\mathcal{R}}\right\|_{0}$. As we only work with the norm of binary vectors, we point that $\|\boldsymbol{a}\|_{0}=\|\boldsymbol{a}\|_{1}$; for simplicity, we omit the subscript. With the partitioning of the sample space into $\mathcal{A}$ and $\mathcal{M}$

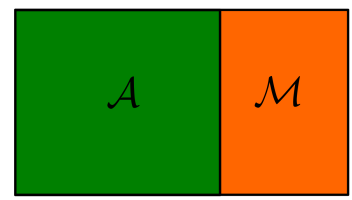

(a)

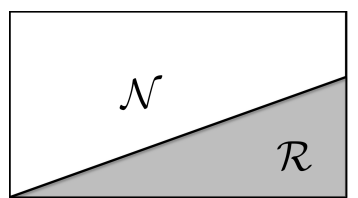

(b)

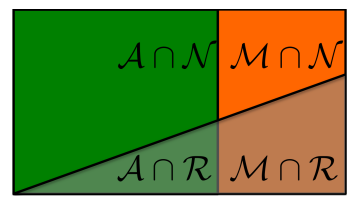

(c)

Figure 1: Partition of the sample space based on the performance of the (a) classification only (partition space $\mathcal{A}$ and $\mathcal{M}$ ); (b) rejection only (partition space $\mathcal{R}$ and $\mathcal{N}$ ); and (c) classification with rejection. Green corresponds to accurately classified samples and orange to misclassified samples. Gray corresponds to rejected samples and white to nonrejected samples.

according to the values of the binary vector $\boldsymbol{a}$, and the partitioning of the sample space into $\mathcal{N}$ and $\mathcal{R}$, we thus partition the sample space as in Fig. 1:

\footnotetext{
${ }^{1}$ We note that $R$ corresponds to a rejector, a function that maps classification into a binary rejection vector, whereas $\mathcal{R}$ denotes a set of samples that are rejected.
} 
- $\mathcal{A} \cap \mathcal{N}$ : samples accurately classified and not rejected; the number of such samples is $|\mathcal{A} \cap \mathcal{N}|=\left\|\boldsymbol{a}_{\mathcal{N}}\right\|$

- $\mathcal{M} \cap \mathcal{N}$ : samples misclassified and not rejected; the number of such samples is $|\mathcal{M} \cap \mathcal{N}|=\left\|\mathbf{1}-\boldsymbol{a}_{\mathcal{N}}\right\|$

- $\mathcal{A} \cap \mathcal{R}$ : samples accurately classified and rejected; the number of such samples is $|\mathcal{A} \cap \mathcal{R}|=\left\|\boldsymbol{a}_{\mathcal{R}}\right\|$

- $\mathcal{M} \cap \mathcal{R}$ : samples misclassified and rejected; the number of such samples is $|\mathcal{M} \cap \mathcal{R}|=\left\|\mathbf{1}-\boldsymbol{a}_{\mathcal{R}}\right\|$

\subsection{Comparing classifiers with rejection}

The comparison of the performance of two rejectors is nontrivial. It depends on the existence of a problem specific cost function that takes in account the trade-off between misclassification and rejection. If a cost function exists, the performance is linked to the comparison of the cost function evaluated on each rejector. However, as previously stated, the design of a problem specific cost function might not be feasible. Let $\rho$ denote the trade-off between rejection and misclassification, thus defining a family of cost functions where a misclassification has a unitary cost, a rejection has a cost of $\rho$. This family of cost functions can be expressed as

$$
|\mathcal{M} \cap \mathcal{N}|+\rho|\mathcal{R}|=\square+\rho
$$

In a probabilistic interpretation, this cost function corresponds to an extended classification risk that takes in account rejection.

Then, there are three general cases where it is possible to perform comparisons between the performance of two rejectors independently of $\rho$ : when 
the number of rejected samples is the same; when the number of accurately classified samples not rejected is the same; and when the number of misclassified samples not rejected is the same. This is true for all values of $\rho$, if we assume that $0 \leq \rho \leq 1$, which is a reasonable assumption as $\rho<0$ would lead to a rejection only problem (all samples rejected), and $\rho>1$ would lead to a classification only problem (no samples are rejected). Let $C$ denote a classifier with an accuracy vector $\boldsymbol{a}$, and $R_{1}$ and $R_{2}$ denote two different rejection mechanisms that partition the sample space in $\mathcal{N}_{R_{1}}, \mathcal{R}_{R_{1}}$ and $\mathcal{N}_{R_{2}}, \mathcal{R}_{R_{2}}$ respectively.

In the following cases we consider the most general concept of outperformance possible, when the cost function of $R_{1}$ is smaller than the cost function of $R_{2}$ for all values of $\rho$, such that $0 \leq \rho \leq 1$.

Equal number of rejected samples

If both rejectors reject the same number of samples, and if rejector $R_{1}$ has a larger number of accurately classified samples than $R_{2}$, then $R_{1}$ outperforms $R_{2}$.



Equal number of nonrejected accurately classified samples

If both rejectors have the same number of accurately classified samples not rejected, and if rejector $R_{1}$ rejects more samples than $R_{2}$, then $R_{1}$ outperforms $R_{2}$.

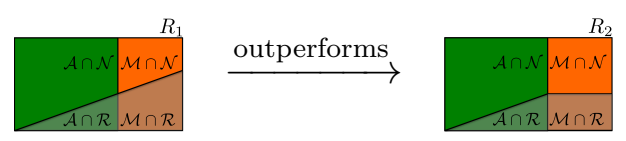




\section{Equal number of nonrejected misclassified samples}

If both rejectors have the same number of misclassified samples not rejected, and if rejector $R_{1}$ rejects fewer samples than $R_{2}$, then $R_{1}$ outperforms $R_{1}$.

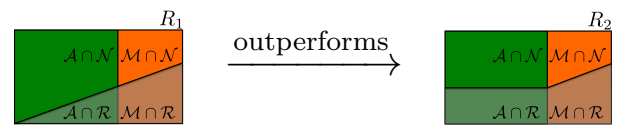

\subsection{Desired properties of performance measures}

The definition of the rejection problem as the partition of the accuracy vector $\boldsymbol{a}$ based on two disjoint supports $\mathcal{N}$ and $\mathcal{R}$ is general and allows us to define desired characteristics for any generic performance measure $\alpha$ that evaluates the performance of classification with rejection.

We start by introducing the rejected fraction $r$, as the ratio of rejected samples versus the overall number of samples,

$$
r=\frac{n-k}{n}=\frac{|\mathcal{R}|}{|\mathcal{R}|+|\mathcal{N}|}=
$$

2.4.1. Property I: Performance measure is a function of the rejected fraction

Given a performance measures $\alpha$ it should be a function of the fraction of rejected samples $r$ :

$$
\alpha=\alpha(r)
$$

2.4.2. Property II: Performance measure is able to compare different rejector mechanisms working at the same rejected fraction

For the same classification $C$, and for two different rejection mechanisms $R_{1}$ and $R_{2}$, the performance measures $\alpha\left(C, R_{1}, r\right)$ and $\alpha\left(C, R_{2}, r\right)$ should be able to compare the rejection mechanisms $R_{1}$ and $R_{2}$ when rejecting the same fraction:

$$
\overbrace{\alpha\left(C, R_{1}, r\right)}^{\text {rejector } R_{1}}>\overbrace{\alpha\left(C, R_{2}, r\right)}^{\text {rejector } R_{2}} \Longleftrightarrow R_{1} \text { outperforms } R_{2} \text {. }
$$


2.4.3. Property III: Performance measure is able to compare different rejector mechanisms working at different rejected fractions

On the other hand, it is also desired that the performance measure be able to compare the performance of different rejection mechanisms $R_{1}$ and $R_{2}$ when they reject different fractions $r_{1}$ and $r_{2}$ :

$$
R_{1} \text { outperforms } R_{2} \Longrightarrow \overbrace{\alpha\left(C, R_{1}, r_{1}\right)}^{\text {rejector } R_{1}}>\overbrace{\alpha\left(C, R_{2}, r_{2}\right)}^{\text {rejector } R_{2}} .
$$

\subsubsection{Property IV: Maximum and minimum values for performance mea-} sures

Any performance measure should achieve its maximum when $\mathcal{N}$ coincides with $\mathcal{A}$ and thus $\mathcal{R}$ with $\mathcal{M}$, corresponding to simultaneously rejecting all misclassified samples and not rejecting any accurately classified samples $\left(\boldsymbol{a}_{\mathcal{N}}=0\right.$ and $\boldsymbol{a}_{\mathcal{R}}=1$ are empty). Similarly, the performance measure should achieve its minimum when $\mathcal{N}$ coincides with $\mathcal{M}$ and $\mathcal{R}$ with $\mathcal{A}$, corresponding to rejecting all accurately classified samples and not rejecting any misclassified samples ( $\boldsymbol{a}_{\mathcal{N}}=1$ and $\boldsymbol{a}_{\mathcal{R}}=0$ are empty).

\section{Performance measures}

We are now ready to define the three performance measures. First, we will show that the nonrejected accuracy, as used extensively in the literature, satisfies all our properties. We will then present two other measures that also satisfy the same properties: classification quality and rejection quality. 


\subsection{Nonrejected accuracy $A$}

The nonrejected accuracy measures the accuracy on the subset of nonrejected samples

$$
A=\frac{\left\|\boldsymbol{a}_{\mathcal{N}}\right\|}{n-k}=\frac{\left\|\boldsymbol{a}_{\mathcal{N}}\right\|}{|\mathcal{N}|}=
$$

The nonrejected accuracy measures the proportion of samples that are accurately classified and not rejected compared to the samples that are not rejected. In a probabilistic interpretation, it is equivalent to the conditional probability of a sample being accurately classified given that it was not rejected.

We can represent the nonrejected accuracy as a function of the rejected fraction,

$$
A=\frac{\left\|\boldsymbol{a}_{\mathcal{N}}\right\|}{|\mathcal{N}|}=\frac{\left\|\boldsymbol{a}_{\mathcal{N}}\right\|}{n(1-r)}=A(r)
$$

satisfying Property I. Properties II, III, and IV are also satisfied; the proof is given in the Appendix.

We note that the maximum and minimum values of the nonrejected accuracy, 1 and 0 respectively, are not necessarily unique. Two different rejectors can have a nonrejected accuracy of 1 if the nonrejected samples are all accurately classified. For example, if rejector $R_{1}$ rejects all misclassified samples and does not reject any accurately classified samples, $\mathcal{R}=\mathcal{M}$, and rejector $R_{2}$ rejects all misclassified samples and some accurately classified samples, $\mathcal{R} \supseteq \mathcal{M}$, both their nonrejected accuracies will be 1 . This is due to the fact that, while the number of nonrejected accurately classified samples decreases, so does the total number of nonrejected samples in the same quantity, thus leading two different situations with equal nonrejected accuracy. 


\subsection{Classification quality $Q$}

The classification quality measures the correct decision making of the classifier-rejector, assessing both the performance of the classifier on the set of nonrejected samples and the performance of the rejector on the set of misclassified samples. This equates to measuring the number of accurately classified samples not rejected $\mathcal{A} \cap \mathcal{N}$ and the number of misclassified samples rejected $\mathcal{M} \cap \mathcal{R}$,

$$
Q=\frac{\left\|\boldsymbol{a}_{\mathcal{N}}\right\|+\left\|\mathbf{1}-\boldsymbol{a}_{\mathcal{R}}\right\|}{|\mathcal{N}|+|\mathcal{R}|}=\frac{\left\|\boldsymbol{a}_{\mathcal{N}}\right\|+\left\|\mathbf{1}-\boldsymbol{a}_{\mathcal{R}}\right\|}{n}=
$$

In a probabilistic interpretation, this is equivalent to the probability of a sample being accurately classified and not rejected or a sample being misclassified and rejected.

To represent the classification quality $Q$ as a function of the fraction of rejected samples $r$, we analyze separately the performance of the classifier on the subset of nonrejected samples and the performance of the rejector on the subset of misclassified samples. The performance of the classifier on the subset of nonrejected samples is the proportion of accurately classified samples not rejected to the total number of samples, which can be easily represented in terms of the nonrejected accuracy as follows,

$$
\frac{\left\|\boldsymbol{a}_{\mathcal{N}}\right\|}{n}=\frac{\left\|\boldsymbol{a}_{\mathcal{N}}\right\|}{n(1-r)}(1-r)=A(r)(1-r) .
$$

The performance of the rejector on the subset of misclassified samples is

$$
\begin{array}{r}
\frac{\left\|\mathbf{1}-\boldsymbol{a}_{\mathcal{R}}\right\|}{n}=\frac{\|\mathbf{1}-\boldsymbol{a}\|-\left\|\mathbf{1}-\boldsymbol{a}_{\mathcal{N}}\right\|}{n}=1-A(0)-\frac{\left\|\mathbf{1}-\boldsymbol{a}_{\mathcal{N}}\right\|}{n}= \\
1-A(0)-\frac{k-\left\|\boldsymbol{a}_{\mathcal{N}}\right\|}{n}=1-A(0)-\frac{k}{n}+\frac{\left\|\boldsymbol{a}_{\mathcal{N}}\right\|}{n}= \\
1-A(0)-(1-r)+A(r)(1-r)=-A(0)+r+A(r)(1-r) .
\end{array}
$$


By combining (10) and (11), we can represent the classification quality as

$$
Q(r)=2 A(r)(1-r)+r-A(0)
$$

satisfying Property I. Properties II, III, and IV are also satisfied; the proof is given in the Appendix.

We note that both the maximum and the minimum values of the classification quality, 1 and 0 respectively, are unique. $Q(r)=1$ describes an ideal rejector that does not reject any of the accurately classified samples and rejects all misclassified samples, $\mathcal{A}=\mathcal{N}$ and $\mathcal{M}=\mathcal{R}$. Conversely, $Q(r)=0$ describes the worst rejector that rejects all the accurately classified samples and does not reject any misclassified sample, $\mathcal{A}=\mathcal{R}$ and $\mathcal{M}=\mathcal{N}$.

We can use the classification as in (12) to compare the proportion of correct decisions between two different rejectors, for different values of rejected fractions. We note that as $Q(0)=A(0)$, we can compare the proportion of correct decisions by using classification with rejection versus the use of no rejection at all.

\subsection{Rejection quality $\phi$}

Finally, we present the rejection quality to evaluate the ability of the rejector to reject misclassified samples. This is measured through the ability to concentrate all misclassified samples onto the rejected portion of samples. The rejection quality is computed by comparing the proportion of misclassified to accurately classified samples on the set of rejected samples with the proportion of misclassified to accurately classified samples on the entire data set,

$$
\phi=\frac{\left\|1-a_{\mathcal{R}}\right\|}{\left\|a_{\mathcal{R}}\right\|} / \frac{\|1-a\|}{\|a\|}=\frac{\square}{\square}
$$


As the rejection quality is not defined when there are no misclassified rejected samples, $\mid \boldsymbol{a}_{\mathcal{R}} \|=0$, we define $\phi \equiv \infty$ if any sample is rejected $|\mathcal{R}|>0$, meaning that no accurately classified sample is rejected and some misclassified samples are rejected, and $\phi \equiv 1$ if no sample is rejected $|\mathcal{R}|=0$. To express the rejection quality as a function of the rejected fraction, we note that, by (1), we can represent the accuracy on the rejected fraction as $\left\|\boldsymbol{a}_{\mathcal{R}}\right\|=\|\boldsymbol{a}\|-\left\|\boldsymbol{a}_{\mathcal{N}}\right\|$, and $\|1-\boldsymbol{a}\|$ as $n(1-A(0))$. This means that

$$
\phi=\frac{r-A(0)+A(r)(1-r)}{A(0)-A(r)(1-r)} \frac{A(0)}{1-A(0)}=\phi(r)
$$

satisfying Property I. Properties II, III, and IV are also satisfied; the proof is given in the Appendix.

Unlike the nonrejected accuracy and the classification quality, the rejection quality is unbounded. A value of $\phi$ greater than one means that the rejector is effectively decreasing the concentration of misclassified samples on the subset of nonrejected samples, thus increasing the nonrejected accuracy.

The minimum value of $\phi$ is 0 , and its maximum is unbounded by construction. Any rejector that only rejected misclassified samples will achieve a $\phi$ value of $\infty$, regardless of not rejecting some misclassified samples.

\section{Quantifying performance of a classifier with rejection}

With the three performance measures defined, we can now compare performance of classifiers with rejection. We illustrate this in Fig. 2, where we consider a general classifier with rejection. In the figure, black circles in the center correspond to a classifier that rejects $20 \%$ of the samples, with a nonrejected accuracy of $62.5 \%$, a classification quality of $65 \%$, and a rejection quality of 3.67; we call that black circle a reference operating point. 


\subsection{Reference operating point, operating point and operating set}

A set of performance measures and the associated rejected fraction $r$ correspond to a reference operating point of the classifier with rejection. Given a reference operating point, we define the operating set as the set of achievable operating points as a function of the rejected fraction. This further means that for each operating point of a classifier with rejection there is an associated operating set.

Any point in the green region of each of the plots in Fig. 2 is an operating point of a classifier with rejection that outperforms the one at the reference operating point (black circle), and any operating point in the orange region is an operating point of a classifier with rejection that is outperformed by the one at the reference operating point (black circle), regardless of the cost function (assuming that the cost of rejection is never greater than the cost of misclassification). In white regions, performance depends on the tradeoff between rejection and misclassification, and is thus dependent of the cost function. The borders of the green and orange regions correspond to the best and worst behaviors, respectively, of classifiers with rejection as compared to the reference operating point. Thus, given the reference operating point, its correspondent operating set is the union of the white regions including the borders.

\subsection{Relative optimality}

To compare the behavior of a classifier with rejection in the white region to that at the reference operating point, we measure how close that classifier is to the green and orange region borders (best/worst behaviors). Let $\beta=0$ denote the curve that corresponds to the middle point between the best and 




(a) Nonrejected accuracy

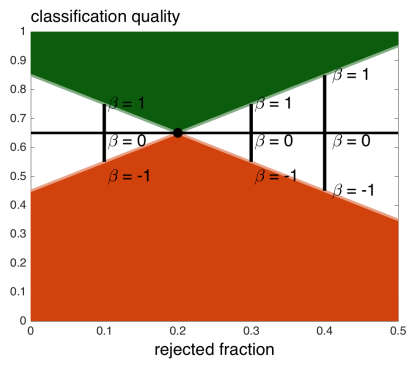

(b) Classification quality

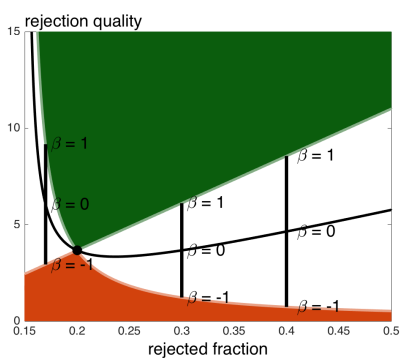

(c) Rejection quality

Figure 2: Performance measures with outperformance (green) underperformance (orange) regions for a reference operating point (black circle). Reference classifier rejects $20 \%$ of the samples and achieves a nonrejected accuracy of $62.5 \%$, classification quality of $65 \%$, and a rejection quality of 3.67. $\beta$ measures correctness of rejection; $\beta=1$ corresponds to the best and $\beta=-1$ to the worst rejection behaviors, respectively.

worst behaviors (black curve in Fig. 2), $\beta=1$ to the best behavior (border with the green region), and $\beta=-1$ to the worst behavior (border with the orange region). We call $\beta$ relative optimality, as it compares the behavior of a classifier with rejection relative to a given reference operating point.

Let us consider a reference operating point defined by a nonrejected accuracy $A_{0}$ at a rejected fraction $r_{0}$; we can now compare the performance at an arbitrary operating point $\left(A_{1}, r_{1}\right)$ with that at a reference operating point $\left(A_{0}, r_{0}\right)$ by computing the relative optimality

$$
\beta= \begin{cases}2 \frac{A_{1}\left(1-r_{1}\right)-A_{0}\left(1-r_{0}\right)}{r_{1}-r_{0}}+1, & \text { if } r_{1}>r_{0}, \\ -2 \frac{A_{1}\left(1-r_{1}\right)-A_{0}\left(1-r_{0}\right)}{r_{1}-r_{0}}-1, & \text { if } r_{1}<r_{0} .\end{cases}
$$

Assuming $r_{1}>r_{0}$, the computation of $\beta$ amounts to computing the relative position of the operating point $\left(A_{1}, r_{1}\right)$ relative to the best and worst rejection behaviors, respectively $\beta=1$ and $\beta=-1$. When from $r_{0}$ to $r_{1}$ only misclassified samples are rejected, then we have that the number of 
accurately classified samples not rejected in both operating points is the same $\left(A_{1}\left(1-r_{1}\right)-A_{0}\left(1-r_{0}\right)=0\right)$, leading to $\beta=1$ in (15). Conversely, when from $r_{0}$ to $r_{1}$ only accurately classified samples are rejected, we have that the number of accurately classified samples decays by $r_{1}-r_{0}$ $\left(A_{1}\left(1-r_{1}\right)-A_{0}\left(1-r_{0}\right)=r_{0}-r_{1}\right)$, leading to $\beta=-1$ in $(15)$.

\subsection{Cost function}

Furthermore, the relative optimality allows us to compare any two operating points of a classifier with rejection taking in account a cost function $L$ which measures the relative cost of rejection versus misclassification. Let us consider the following generic cost function

$$
L_{\rho}\left(\hat{y}_{i}^{R}\right)= \begin{cases}0, & \hat{y}_{i}^{R} \text { accurately classified and not rejected } \\ 1, & \hat{y}_{i}^{R} \text { misclassified and not rejected } \\ \rho, & \hat{y}_{i}^{R} \text { rejected }\end{cases}
$$

where $\rho$ is the cost of rejection and represents the trade-off between rejection and misclassification. We can also compute the cost function (16) at an operating point $(A, r)$ as a function of the nonrejected accuracy and the rejected fraction as $L_{\rho}(A, r)=(1-r)(1-A) n+\rho r n$. We now connect the concept of relative optimality with the generic cost function $L$ as follows.

Theorem 1. For an operating point $\left(A_{1}, r_{1}\right)$ with a relative optimality $\beta$ relative to the reference operating point $\left(A_{0}, r_{0}\right)$, and $r_{1}>r_{0}$,

$$
\operatorname{sgn}\left(\Delta L_{\rho}\right)=\operatorname{sgn}\left(L_{\rho}\left(A_{0}, r_{0}\right)-L_{\rho}\left(A_{1}, r_{1}\right)\right)=\operatorname{sgn}\left(\frac{\beta+1}{2}-\rho\right),
$$

where $\Delta L_{\rho}$ is the difference between the cost function at the reference operating point $L_{\rho}\left(A_{0}, r_{0}\right)$ and the cost function at the operating point $L_{\rho}\left(A_{1}, r_{1}\right)$. 
Proof. Let $r_{1}>r_{0}$; then we have that the cost function at a generic operating point $(A, r)$ is

$$
L_{\rho}(A, r)=(1-r)(1-A) n+\rho r n,
$$

as we have $(1-r)(1-A) n$ misclassified samples, $(1-r) A n$ accurately classified samples, and $r n$ rejected samples, and thus

$$
\begin{aligned}
\Delta L_{\rho} & =n\left(\left(1-r_{0}\right)\left(1-A_{0}\right)+\rho r_{0}-\left(1-r_{1}\right)\left(1-A_{1}\right)-\rho r_{1}\right) \\
& =n\left(r_{1}-r_{0}-\left(1-r_{0}\right) A_{0}+\left(1-r_{1}\right) A_{1}+\rho\left(r_{0}-r_{1}\right)\right) .
\end{aligned}
$$

On the other hand, from (15), we have that

$$
A_{1}\left(1-r_{1}\right)-A_{0}\left(1-r_{0}\right)=\frac{\beta-1}{2}\left(r_{1}-r_{0}\right)
$$

By combining (19) and (20), we have that

$$
\Delta L_{\rho}=n\left(r_{1}-r_{0}\right)\left(\frac{\beta+1}{2}-\rho\right)
$$

Because $r_{1}-r_{0}$ and $n$ are positive, $\Delta L_{\rho}$ and $(\beta+1) / 2-\rho$ have the same sign.

The previous discussion allows us to compare a classifier with rejection $R_{1}$ to the reference operating point $R_{0}$ as follows. Let the operating point $\left(A_{1}, r_{1}\right)$ be at relative optimality $\beta$ with respect to the reference operating point $\left(A_{0}, r_{0}\right)$. Then,

$$
\begin{cases}L_{\rho}\left(A_{1}, r_{1}\right)<L_{\rho}\left(A_{0}, r_{0}\right), & \text { for } \rho<(\beta+1) / 2 \\ L_{\rho}\left(A_{1}, r_{1}\right) \geq L_{\rho}\left(A_{0}, r_{0}\right), & \text { for } \rho \geq(\beta+1) / 2 .\end{cases}
$$




\subsection{Performance measures}

We can consider the classifier with rejection as two coupled classifiers if we considered the rejector $R$ to be a binary classifier on the output $\hat{y}$ of the classifier $C$, assigning to each sample a rejected or nonrejected label. Ideally, $R$ should classify as rejected all samples misclassified by $C$ and classify as nonrejected all the samples accurately classified by $C$.

In this binary classification formulation, the classification quality $Q$ becomes the accuracy of the binary classifier $R$, the accuracy of the nonrejected samples $A$ becomes the precision (positive predictive value) of the binary classifier $R$, and the rejection quality $\phi$ becomes the positive likelihood ratio (the ratio between the true positive rate and the false positive rate) of the binary classifier $R$. The rejected fraction becomes the ratio between the number of samples classified as rejected and the total number of samples.

As we are able to reconstruct the confusion matrix from the triplet $(A(r), Q(r), r)$, we are thus able to show that the triplet is sufficient to describe the behavior of the rejector. This means that this formulation allows us to show that the triplet $(A(r), Q(r), r)$ completely specifies the behavior of the rejector by relating the triplet to the confusion matrix associated with the binary classifier $R$.

Theorem 2. The set of measures $(A(r), Q(r), r)$ completely specifies the behavior of the rejector.

Proof. Let us consider the following confusion matrix associated with $R$ :

$$
\left[\begin{array}{cc}
|\mathcal{A} \cap \mathcal{N}| & |\mathcal{M} \cap \mathcal{N}| \\
|\mathcal{A} \cap \mathcal{R}| & |\mathcal{M} \cap \mathcal{R}|
\end{array}\right],
$$


where $n$ denotes the total number of samples, $|\mathcal{A} \cap \mathcal{N}| / n$ the number of samples accurately classified and not rejected, $|\mathcal{M} \cap \mathcal{N}|$ the number of samples misclassified but not rejected, $|\mathcal{A} \cap \mathcal{R}|$ the number of samples accurately classified but rejected, and $|\mathcal{M} \cap \mathcal{R}|$ the number of samples misclassified and rejected. Given that $n$ binary classifications classified $n$ samples, the confusion matrix associated with $R$ can be uniquely obtained from the following full rank system:

$$
\left[\begin{array}{c}
|\mathcal{A} \cap \mathcal{N}| \\
|\mathcal{M} \cap \mathcal{N}| \\
|\mathcal{A} \cap \mathcal{R}| \\
|\mathcal{M} \cap \mathcal{R}|
\end{array}\right]=n\left[\begin{array}{cccc}
0 & 0 & 0 & 1 \\
1 & -1 & 0 & -1 \\
0 & 0 & 1 & 1 \\
0 & 1 & -1 & 1
\end{array}\right]\left[\begin{array}{c}
1 \\
r \\
Q(r) \\
A(r)(1-r)
\end{array}\right]
$$

This full-rank system corresponds to the representation of the elements of the confusion matrix associated with $R$ as a linear combination of: the number of samples $n$, the number of rejected samples $n r$, the number, the number of samples that are accurately classified and not rejected or misclassified and rejected $n Q(r)$, and the number of samples that are accurately classified and not rejection $n A(r)(1-r)$. Therefore, as the set of measures and the confusion matrix are related by a full-rank system, the set of measures $(A(r), Q(r), r)$ completely specifies describes the behavior of the rejector.

\subsection{Comparing performance of classifiers with rejection}

Given a classifier $C$ and two rejectors $R_{1}$ and $R_{0}$, with $r_{1}>r_{0}$, and a cost function with a rejection-misclassification trade-off $\rho$, we can now compare the performance of classifiers with rejection.

Rejector $R_{1}$ outperforms $R_{0}$ when the following equivalent conditions are 
satisfied:

$A_{R_{1}}\left(r_{1}\right)>A_{R_{0}}\left(r_{0}\right) \frac{1-r_{0}}{1-r_{1}}+(\rho-1) \frac{r_{1}-r_{0}}{1-r_{1}} \Longleftrightarrow Q_{R_{1}}\left(r_{1}\right)>Q_{R_{0}}\left(r_{0}\right)+(2 \rho-1)\left(r_{1}-r_{0}\right)$.

Rejector $R_{0}$ outperforms $R_{1}$ when the following equivalent conditions are satisfied:

$A_{R_{1}}\left(r_{1}\right)<A_{R_{0}}\left(r_{0}\right) \frac{1-r_{0}}{1-r_{1}}+(\rho-1) \frac{r_{1}-r_{0}}{1-r_{1}} \Longleftrightarrow Q_{R_{1}}\left(r_{1}\right)<Q_{R_{0}}\left(r_{0}\right)+(2 \rho-1)\left(r_{1}-r_{0}\right)$.

Rejectors $R_{0}$ and $R_{1}$ are equivalent in terms of performance when the following equivalent conditions are satisfied:

$A_{R_{1}}\left(r_{1}\right)=A_{R_{0}}\left(r_{0}\right) \frac{1-r_{0}}{1-r_{1}}+(\rho-1) \frac{r_{1}-r_{0}}{1-r_{1}} \Longleftrightarrow Q_{R_{1}}\left(r_{1}\right)=Q_{R_{0}}\left(r_{0}\right)+(2 \rho-1)\left(r_{1}-r_{0}\right)$.

The proof is given in the Appendix.

\section{Experimental results}

The three proposed performance measures can easily be applied to experimental situations

To illustrate the use of the proposed performance measures, we apply them to the analysis of the performance of classifiers with rejection applied to synthetic and real data. We use a simple synthetic problem to motivate the problem of classification with rejection and to serve as a toy example.

We then focus on the application of classification with rejection to pixelwise hyperspectral image classification $[7,8]$, which is prone to the effects of small and nonrepresentative training sets, meaning that the classifiers might not be equipped to deal with all existing classes, due to the potential presence of unknown classes. Classification with rejection is an interesting avenue for hyperspectral image classification as the need to accurately classify the samples is greater than the need to classify all samples. 


\subsection{Synthetic data}

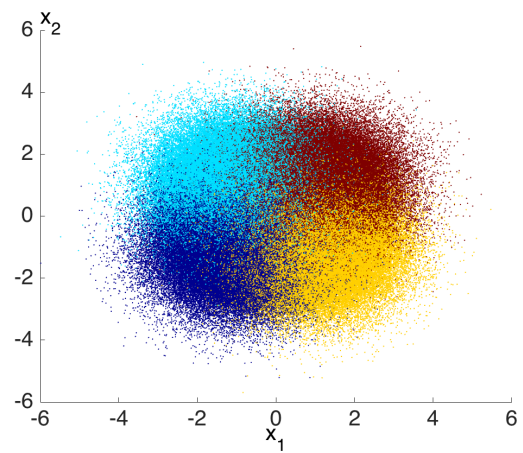

(a) Observed data

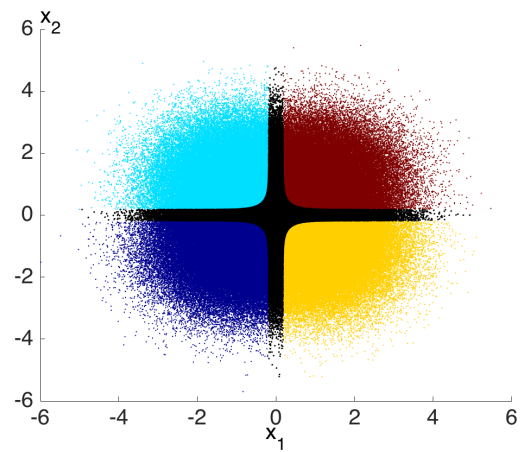

(c) Class. $20 \%$ rej.



(b) Classification no rejection

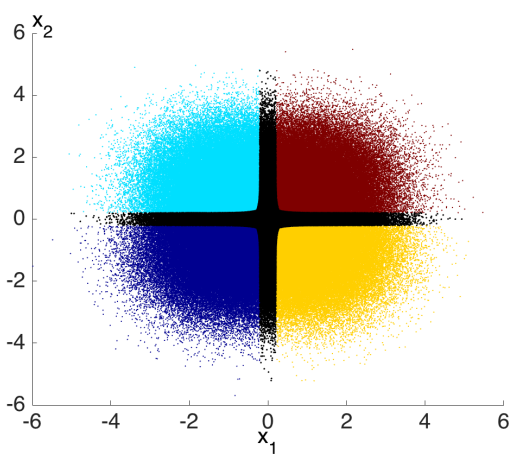

(d) Class. $20 \%$ rej. breaking ties

Figure 3: Synthetic data example. Samples of four equally likely Gaussians with equal covariance (identity covariance matrix) and significant overlap (centered at $( \pm 1, \pm 1)$ ), classified with rejection (in black). (a) Observed data, (b) classification with no rejection, (c) classification with $20 \%$ rejection using maximum probability rejector, and (d) classification with $20 \%$ rejection using breaking ties rejector. The differences between the two rejectors are clear near the origin.

As a toy example, we consider a classification problem consisting of four two-dimensional Gaussians with the identity matrix as a covariance matrix 
and centers at $( \pm 1, \pm 1)$. The Gaussians overlap significantly, as shown in

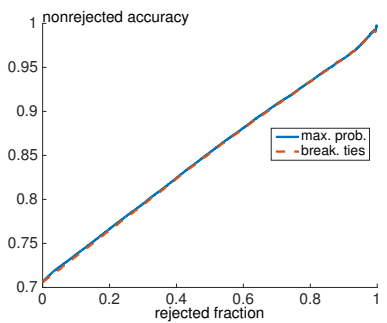

(a) Nonrejected accuracy



(b) Classification quality

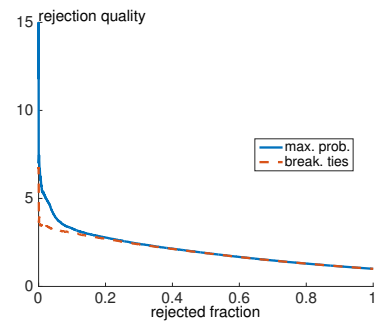

(c) Rejection quality

Figure 4: Performance measures as a function of the rejected fraction for the synthetic example and the maximum probability rejector (solid blue line), and the breaking ties (dashed red line).

Fig.3(a). This results in a simple classification decision using the Maximum Likelihood criterion: for each sample, assign the label of the class with the closest center as in Fig.3(b).

We illustrate our performance measures by comparing two simple rejection mechanisms: (1) maximum probability rejector, which, given a classifier and a rejected fraction, rejects the fraction of samples with lower probability; and (2) breaking ties rejector, which, given a classifier and a rejected fraction, rejects the fraction of samples with lower difference between the highest and second-highest class probabilities.

In Fig.4, we can see the performance measures computed for all possible rejected fractions for each of the two rejectors. It is clear that the with the accuracy-rejection curves alone, as shown in Fig.4(a), we are not able to single out any operating point of the classifier with rejection. On the other hand, with the classification quality in Fig.4(b), we can identify where the 
rejector is maximizing the number of correct decisions, and for which cases having a reject option outperforms not having rejection. As illustrated in Fig.4(c), the rejection quality provides an easy way to discriminate between two different rejectors, as it focuses on the analysis of the ratios of correctly classified to incorrectly classified samples on the set of rejected samples.

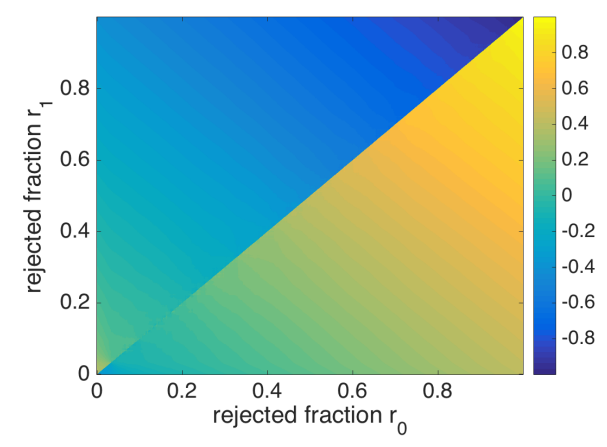

(a) Maximum probability rejector

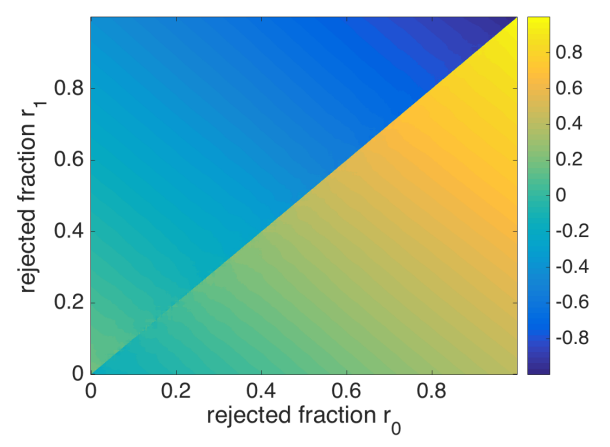

(b) Breaking ties rejector

Figure 5: Relative optimality computed for all possible pairs of operating points of (a) maximum probability rejector and (b) breaking ties rejector

The relative optimality plots for both rejectors are present in Fig. 5. For each possible operating point of the rejector, for simplicity defined only by the rejected fraction, we compute the relative optimality of all other operating points of the rejector. We note that, for both rejectors, the operating point that corresponds to the maximum classification quality, has a nonnegative relative optimality with regards to all other operating points. This relative optimality plot is of particular interest for parameter selection. 


\subsection{Hyperspectral image data}

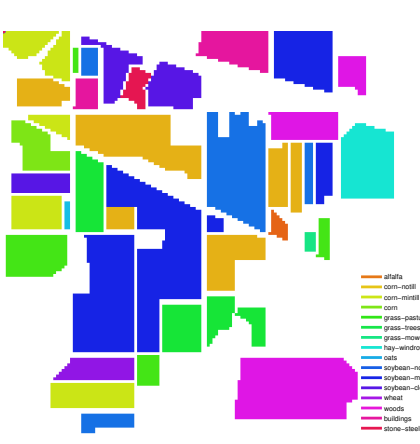

(a) Ground truth

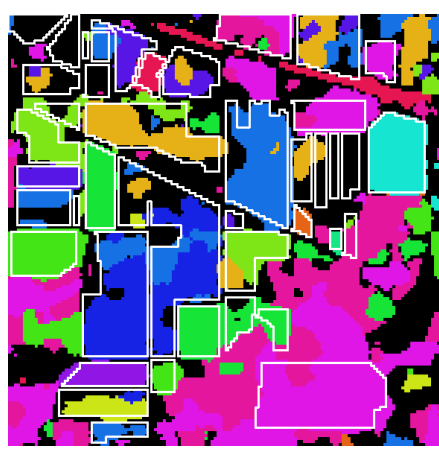

(b) SegSALSA-JCR

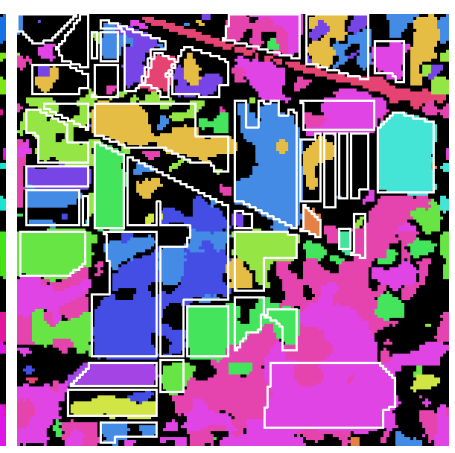

(c) SegSALSA-SCR

Figure 6: Indian Pine scene. (a) ground truth, (b) classification with context and rejection with SegSALSA-JCR, and (c) classification with context and rejection with SegSALSASCR.

In hyperspectral image classification, the use of context through the form of spatial priors is widespread, providing significant performance improvements [54]. This means that after classification, a computationally expensive procedure is applied to classifier output to take into account contextual effects. The use of accuracy-rejection curves might not be feasible. Changes in the rejected fraction often imply a computationally expensive context computation procedure. Thus, due to the joint use of context and rejection, and the high computational costs associated with context, this is a perfect environment for the use of the performance measures. We use the algorithms for hyperspectral image classification with context and rejection presented in [55] in their joint (JCR) and in their sequential (SCR), versions, respectively. Both JCR and SCR are based on SegSALSA (Segmentation by Split Augmented Lagrangian Shrinkage Algorithm). SegSALSA consists of a soft supervised classifier assigning a class probability to each pixel of the 




(a) Nonrejected accuracy



(b) Classification quality

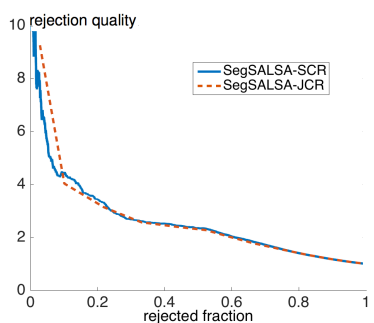

(c) Rejection quality

Figure 7: Performance measures as a function of the rejected fraction for the Indian Pine scene and the SegSALSA-JCR rejector (dashed red line), and SegSALSA-SCR rejector (solid blue line).

image, followed by the application of context through the computation of the marginal maximum a posteriori of a continuous hidden field. For more details on the SegSALSA, see [56].

In the JCR version, rejection is introduced as an extra class that models the probability of classifier failure, considered constant for all the pixels of the hyperspectral image, leading to a uniform weighting of the samples. The higher the probability of failure, the larger the rejected fraction. However, it is not possible to define a priori the amount of rejected fraction obtained, and any change in the value of rejected fraction implies the recomputation of the SegSALSA algorithm.

In the SCR version, rejection is obtained from an approximation of the problem of joint computation of context and rejection. The ordering of each pixel according to its confidence is obtained from the hidden field, following a sequential approach by computing context first and rejection last.

We apply the SegSALSA-JCR and the SegSALSA-SCR to the classifi- 
cation of a well known benchmark image in the hyperspectral community, the AVIRIS Indian Pine scene ${ }^{2}$, as shown in Fig. 6. The scene consists of a $145 \times 145$ pixel section with 200 spectral bands (the water absorption bands are removed) and contains 16 nonmutually exclusive classes.

Following the approach in [55], the class models are learned using a sparse logistic regression with a training set composed of 10 samples per class, and, for the joint approach, perform a parameter sweep on the probability of classifier failure, obtaining various operating points of the JCR rejector. For the SCR rejector, as we define a posteriori the rejected fraction, obtaining operating points of the SCR rejector is simply obtained by rejecting the fraction of pixels with the least amount of confidence. See [55] for a detailed explanation of the JCR and SCR schemes for rejection with context.

As seen in Fig. 7, it is clear that, by looking at the accuracy rejection curves alone, it is trivial to compare the performance of the two rejectors when working at the same rejected fraction. However, we cannot draw any conclusions on which is the best operating point of each rejector, or how they compare to each other. By looking at the classification quality, it is clear where the maximum number of correct decisions is made for each of the rejectors, and by looking at the rejection quality we can observe that there is a significant improvement with reject options for lower values of the rejected fraction.

Fig. 8 shows the relative optimality between each pair of operating points for each of the rejectors. Using (17), for a given reference operating point and

\footnotetext{
${ }^{2}$ We thank Prof. Landgrebe at Purdue University for providing the AVIRIS Indian Pines scene to the community.
} 


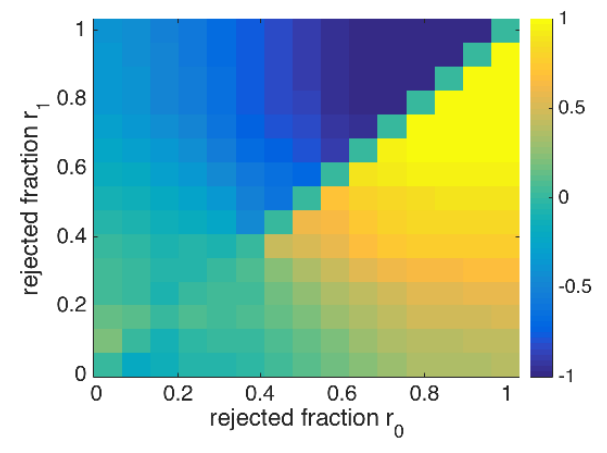

(a) Relative optimality JCR

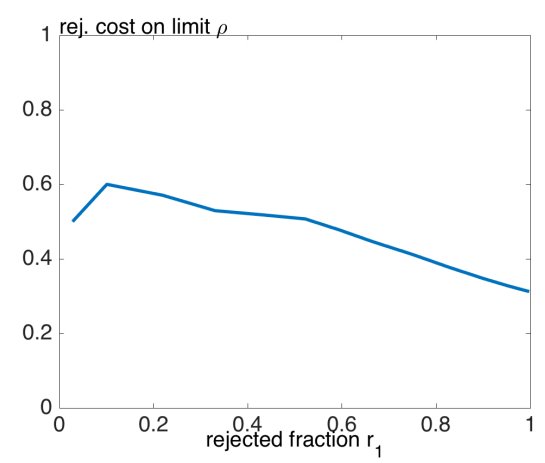

(c) Minimum rejection cost JCR

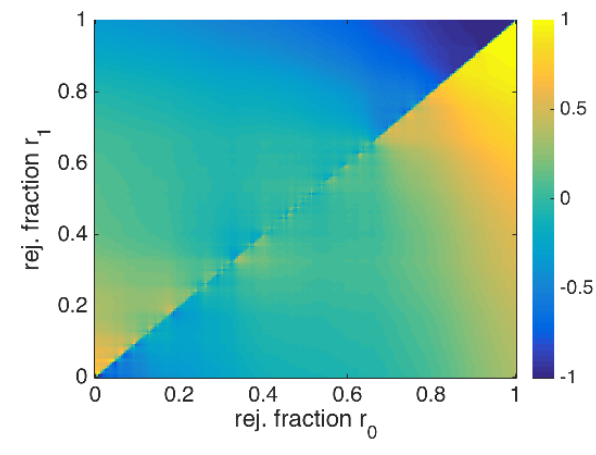

(b) Relative optimality SCR



(d) Minimum rejection cost SCR

Figure 8: Relative optimality computed for all possible pairs of operating points of (a) JCR and (b) SCR rejectors, and minimum value of $\rho$ in the cost function (16), for (c) JCR and (d) SCR rejectors, such that it is better not to reject $\left(r_{0}=0\right)$, for each operating point.

for any test operating point, we can obtain the minimum value of $\rho_{0}$ in the cost function (16) such that the cost function at the test operating point is smaller than the cost function at the reference operating point. This means that, for any cost function with $\rho<\rho_{0}$, the test operating point is better than the reference operating point. We perform such analysis in Fig. 8, where we set the reference operating point as $r_{0}=0$, meaning no rejection. For each 
possible value of rejected fraction $r_{1}$, we then test what the minimum value of the $\rho$ such that no rejection is a better option than rejecting a fraction $r_{1}$ would be, according to the operating points defined by the two rejectors.

\subsection{Adrenal tumor classification}

To illustrate the applicability of the proposed performance measures, we apply them to the evaluation of the performance of the two proposed different rejection mechanisms $[57,58]$ for classification with rejection of adrenal tumors [59, 60]: a global rejection mechanism [57], and a local rejection mechanism [58]. In both cases, the classifier is based on the generalized matrix learning vector quantization (GMLVQ) [61], and the rejection mechanisms are based on the relative similarity (RelSim) measure $[62,57]$ which is inherently associated with the cost function of the GMLVQ classifier.

From Fig. 9, it is clear that whereas the ARC, represented by the nonrejected accuracy-rejection curve in Fig. 9 (a), can compare both rejectors when working at the same rejected fraction, there is no identification of interesting operating points. The analysis of the classification quality in Fig. 9 (b), shows that in both rejectors, the maximum proportion of correct decisions is achieved when no rejection is performed. This is very common in situations where the accuracy of classifier achieves a high level, as the effort required to the rejector to correctly separate misclassified samples from accurately classified samples is significantly increased. With effect, it can be seen that, even though the rejection quality in Fig. 9 (c) achieves high levels for smaller rejected fractions (smaller than 10\%), such values are not high enough to lead to an increase on the classification quality. Finally, we can observe in Fig. 9 (d) that, when compared to the option of not rejecting, 


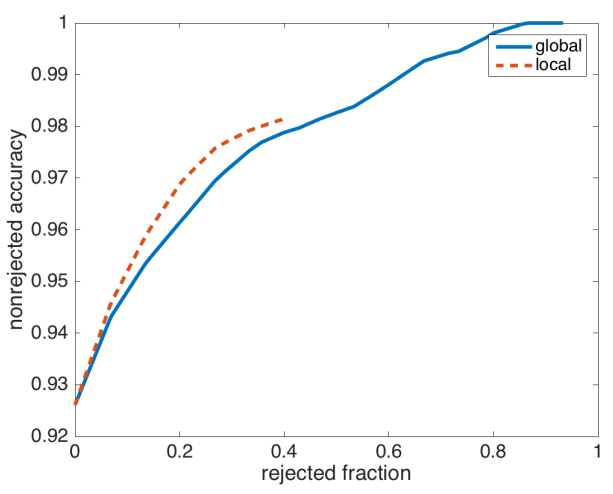

(a) Nonrejected accuracy

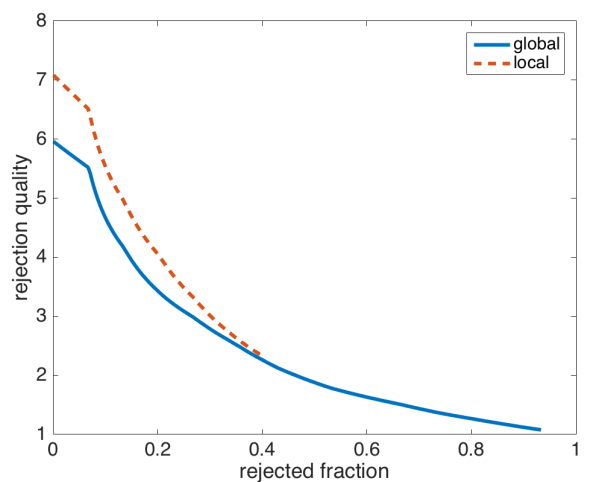

(c) Rejection quality



(b) Classification quality

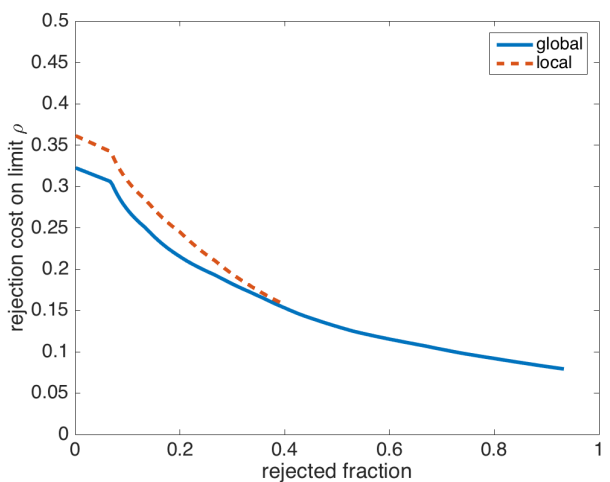

(d) Minimum rejection cost

Figure 9: Performance measures $(a, b, c)$ as a function of the rejected fraction for the rejection mechanisms in $[57,58]$ for the average of 1000 runs and minimum rejection cost (d) such that the operating point outperforms the no rejection situation.

rejection is a feasible alternative, for values of the rejection cost $\rho \leq 0.3$, for all possible rejected fractions smaller than $10 \%$ for the local rejection mechanism and for all possible rejected fraction smaller than $7 \%$ for the global rejection mechanism.

The advantages of an extended set of measures for the evaluation of the 
performance of classifiers with rejection, and the increased breath of analysis of the set of proposed measures are illustrated in this example.

\section{Conclusions}

We introduced a set of measures to quantify performance of classifiers with rejection. We then applied these performance measures to classifiers with rejection on both synthetic and real-world (hyperspectral image) data. Furthermore, we connected the performance measures presented with general cost functions through the concept of relative optimality.

Whereas the use of ARC and based measures is relevant when the goal is the maximization of the nonrejected accuracy for a given rejected fraction or the minimization of the rejected fraction for a given nonrejected accuracy, there are interesting conclusions about the behavior of classification systems with rejection that can be drawn by the use of the concepts of classification quality, rejection quality, and relative optimality.

The proposed measures can easily be used for the evaluation of performance of existing classification systems with rejection, as they only require access to the overall accuracy of the classification system, rejected fraction, and fraction of correctly classified samples on the subset of nonrejected samples (nonrejected accuracy). The performance measures proposed on this paper can easily be extended to account for class-specific rejection costs, this is, to consider a vector of rejection costs $\rho$ instead of a single rejection cost equal for all the classes $\rho$. The class-specific versions of the performance measures can easily be derived, such as the class-specific values of relative optimality. 


\section{Acknowledgments}

The authors would like to thank Lydia Fischer for providing the global and the local rejection using the RelSim certainty measure and the GMLVQ as classifier for the adrenal data. The authors thank the helpful suggestions of the anonymous reviewers. The authors gratefully acknowledge support from the Portuguese Science and Technology Foundation under projects UID/EEA/50008/2013, PTDC/EEIPRO/1470/2012, the Portuguese Science and Technology Foundation and the CMU-Portugal (ICTI) program under grant SFRH/BD/51632/2011, NSF through award 1017278, and the CMU CIT Infrastructure Award.

\section{References}

[1] B. Hanczar, E. Dougherty, Classification with reject option in gene expression data, Bioinformatics 24 (17) (2008) 1889-1895.

[2] C. Botella, J. Ferré, R. Boqué, Classification from microarray data using probabilistic discriminant partial least squares with reject option, Talanta 80 (1) (2009) 321-328.

[3] A. Neto, R. Sousa, G. Barreto, J. Cardoso, Diagnostic of pathology on the vertebral column with embedded reject option, in: Iberian Conference on Pattern Recognition and Image Analysis, Springer, 2011, pp. $588-595$.

[4] J. Quevedo, A. Bahhamonde, M. Pérez-Enciso, O. Luaces, Disease liability prediction from large scale genotyping data using classifiers with a reject option, IEEE/ACM Trans. on Comp. Biology and Bioinformatics (TCBB) 9 (1) (2012) $88-97$. 
[5] F. Condessa, J. Bioucas-Dias, C. Castro, J. Ozolek, J. Kovačević, Classification with rejection option using contextual information, Proc. IEEE Int. Symp. Biomed. Imag. (2013) 1340-1343.

[6] G. Giacinto, F. Roli, L. Bruzzone, Combination of neural and statistical algorithms for supervised classification of remote-sensing images, Patt. Rec. Lett. 21 (5) (2000) 385-397.

[7] F. Condessa, J. Bioucas-Dias, J. Kovačević, Robust hyperspectral image classification with rejection fields, IEEE GRSS Workshop on Hyperspectral Image and Sign. Proc.: Evo. in Remote Sens. (WHISPERS'15) PP (2015) 1-4.

[8] F. Condessa, J. Bioucas-Dias, J. Kovačević, Supervised hyperspectral image classification with rejection, IEEE Geosci. and Remote Sens. Symp. (IGARSS'15) (2015) 2600 - 2603.

[9] D. Mesquita, L. Rocha, J. Gomes, A. Neto, Classification with reject option for software defect prediction, Applied Soft Computing (2016) $1-9$.

[10] J. Navarro-Cerdan, J. Arlandis, R. Llobet, J. Perez-Cortes, Batchadaptive rejection threshold estimation with application to ocr postprocessing, Expert Systems with Applications 42 (21) (2015) 8111-8122.

[11] G. Fumera, I. Pillai, F. Roli, Classification with reject option in text categorisation systems, Proc. 12th Int. Conf. on Image Analysis and Proc. (2003) $582-587$. 
[12] G. Fumera, I. Pillai, F. Roli, A two-stage classifier with reject option for text categorisation, in: Joint IAPR International Workshops on Statistical Techniques in Pattern Recognition (SPR) and Structural and Syntactic Pattern Recognition (SSPR), Springer, 2004, pp. 771-779.

[13] N. Hatami, C. Chira, Classifiers with a reject option for early time-series classification, in: Computational Intelligence and Ensemble Learning (CIEL), 2013 IEEE Symposium on, IEEE, 2013, pp. 9-16.

[14] Y. Zhang, B. Zhang, F. Coenenz, W. Lu, Highly reliable breast cancer diagnosis with cascaded ensemble classifiers, in: The 2012 International Joint Conference on Neural Networks (IJCNN), IEEE, 2012, pp. 1-8.

[15] A. Vailaya, M. Figueiredo, A. Jain, H. Zhang, Image classification for context-based indexing, IEEE Trans. Image Proc. 10 (1) (2001) 117 130.

[16] R. Huber, H. Ramoser, K. Mayer, H. Penz, M. Rubik, Classification of coins using an eigenspace approach, Patt. Rec. Lett. 26 (1) (2005) $61-75$.

[17] A. Payne, S. Singh, Indoor vs. outdoor scene classification in digital photographs, Patt. Rec. 38 (10) (2005) 1533-1545.

[18] B. Dubuisson, M. Masson, A statistical decision rule with incomplete knowledge about classes, Pattern recognition 26 (1) (1993) 155-165.

[19] R. Muzzolini, Y.-H. Yang, R. Pierson, Classifier design with incomplete knowledge, Pattern Recognition 31 (4) (1998) 345-369. 
[20] H. Mouchere, E. Anquetil, A unified strategy to deal with different natures of reject, in: 18th International Conference on Pattern Recognition (ICPR'06), Vol. 2, IEEE, 2006, pp. 792-795.

[21] B. Hanczar, M. Sebag, Combination of one-class support vector machines for classification with reject option, in: Joint European Conference on Machine Learning and Knowledge Discovery in Databases, Springer, 2014, pp. 547-562.

[22] W. Homenda, M. Luckner, W. Pedrycz, Classification with rejection: concepts and evaluations, in: Knowledge, Information and Creativity Support Systems: Recent Trends, Advances and Solutions, Springer, 2016, pp. 413-425.

[23] L. Mascarilla, C. Frelicot, A class of reject-first possibilistic classifiers, in: IFSA World Congress and 20th NAFIPS International Conference, 2001. Joint 9th, Vol. 2, IEEE, 2001, pp. 743-747.

[24] C. Frélicot, L. Mascarilla, A third way to design pattern classifiers with reject options, in: Fuzzy Information Processing Society, 2002. Proceedings. NAFIPS. 2002 Annual Meeting of the North American, IEEE, 2002, pp. 395-399.

[25] L. Mascarilla, C. Frélicot, Reject strategies driven combination of pattern classifiers, Pattern Analysis \& Applications 5 (2) (2002) 234-243.

[26] L. Mascarilla, M. Berthier, C. Frélicot, A k-order fuzzy or operator for pattern classification with k-order ambiguity rejection, Fuzzy Sets and Systems 159 (15) (2008) 2011-2029. 
[27] H. Le Capitaine, C. Frélicot, A new fuzzy 3-rules pattern classifier with reject options based on aggregation of membership degrees, in: 12th International Conference on Information Processing and Management of Uncertainty in Knowledge-Based Systems, 2008, pp. 473-480.

[28] H. Le Capitaine, C. Frelicot, A family of measures for best top-n classselective decision rules, Pattern Recognition 45 (1) (2012) 552-562.

[29] H. Le Capitaine, A unified view of class-selection with probabilistic classifiers, Pattern Recognition 47 (2) (2014) 843-853.

[30] C. K. Chow, On optimum recognition error and reject tradeoff, IEEE Trans. Inform. Th. 16 (1) (1970) 41-46.

[31] M. Wegkamp, Lasso type classifiers with a reject option, Electronic Journ. of Stat. (2007) $155-168$.

[32] P. Bartlett, M. Wegkamp, Classification methods with reject option using a hinge loss, Journ. Machine Learning Research 9 (2008) 1823 1840.

[33] M. Yuan, M. Wegkamp, Classification methods with reject option based on convex risk minimization, Journ. Machine Learning Research 11 (2010) $111-130$.

[34] G. Fumera, F. Roli, Support vector machines with embedded reject option, Proc. Int. Workshop on Patt. Rec. with Support Vector Machines (SVM2002), Niagara Falls (2002) 68- 82. 
[35] F. Tortorella, Reducing the classification cost of support vector classifiers through an roc-based reject rule, Pattern Analysis and Applications 7 (2) (2004) 128-143.

[36] Y. Grandvalet, A. Rakotomamonjy, J. Keshet, S. Canu, Support vector machines with a reject option, Advances in Neural Inf. Proc. Systems (2009) $537-544$.

[37] M. Wegkamp, M. Yuan, Support vector machines with a reject option, Bernoulli 17 (4) (2011) 1368 - 1385.

[38] I. Pillai, G. Fumera, F. Roli, Multi-label classification with a reject option, Patt. Recogn. 46 (8) (2013) 2256 - 2266.

[39] P. Foggia, C. Sansone, F. Tortella, M. Vento, Multiclassification; reject criteria for the bayesian combiner, Patt. Recogn. 32 (8) (1999) 1435 1447.

[40] D. M. Tax, R. P. Duin, Growing a multi-class classifier with a reject option, Patt. Rec. Lett. 29 (10) (2008) 1565-1570.

[41] H. Ramaswamy, A. Tewari, S. Agarwal, Consistent algorithms for multiclass classification with a reject option, arXiv preprint arXiv:1505.04137.

[42] G. Fumera, F. Roli, G. Giacinto, Reject option with multiple thresholds, Patt. Recogn. 33 (12) (2000) 2099 - 2101.

[43] G. Fumera, F. Roli, Analysis of error-reject trade-off in linearly combined multiple classifiers, Patt. Recogn. 37 (6) (2004) 1245 - 1265. 
[44] M. Nadeem, J. Zucker, B. Hanczar, Accuracy-rejection curves (ARCs) for comparing classification methods with a reject option., Machine Learning in Systems Biology (2010) 65-81.

[45] R. Sousa, J. Cardoso, The data replication method for the classification with reject option, AI Communications 26 (3) (2013) 281 - 302.

[46] R. Herbei, M. Wegkamp, Classification with reject option, Canadian Journ. of Stat. 34 (4) (2006) $709-721$.

[47] P. Simeone, C. Marrocco, F. Tortorella, Design of reject rules for ecoc classification systems, Pattern Recognition 45 (2) (2012) 863-875.

[48] I. Pillai, G. Fumera, F. Roli, A classification approach with a reject option for multi-label problems, in: International Conference on Image Analysis and Processing, Springer, 2011, pp. 98-107.

[49] T. Landgrebe, D. Tax, P. Paclík, R. Duin, The interaction between classification and reject performance for distance-based reject-option classifiers, Patt. Rec. Lett. 27 (8) (2006) 908 - 917.

[50] F. Tortorella, An optimal reject rule for binary classifiers, in: Joint IAPR International Workshops on Statistical Techniques in Pattern Recognition (SPR) and Structural and Syntactic Pattern Recognition (SSPR), Springer, 2000, pp. 611-620.

[51] F. Tortorella, A roc-based reject rule for dichotomizers, Pattern Recognition Letters 26 (2) (2005) 167-180. 
[52] T. Pietraszek, On the use of roc analysis for the optimization of abstaining classifiers, Machine Learning 68 (2) (2007) 137-169.

[53] C. Santos-Pereira, A. Pires, On optimal reject rules and roc curves, Pattern Recognition Letters 26 (7) (2005) 943-952.

[54] J. Bioucas-Dias, A. Plaza, G. Camps-Valls, P. Scheunders, N. Nasrabadi, J. Chanussot, Hyperspectral remote sensing data analysis and future challenges, IEEE Geosci. Remote Sens. Magazine 1 (2) (2013) 6 - 36.

[55] F. Condessa, J. Bioucas-Dias, J. Kovačević, Supervised hyperspectral image classification with rejection, IEEE Journ. of Sel. Top. in Applied Earth Observations and Remote Sens. PP (2016) 1-14.

[56] F. Condessa, J. Bioucas-Dias, J. Kovačević, Supervised hyperspectral image segmentation: a convex formulation using hidden fields, IEEE GRSS Workshop on Hyperspectral Image and Sign. Proc.: Evo. in Remote Sens. (WHISPERS'14) PP (2014) 1-4.

[57] L. Fischer, B. Hammer, H. Wersing, Efficient rejection strategies for prototype-based classification, Neurocomputing 169 (2015) 334 - 342.

[58] L. Fischer, B. Hammer, H. Wersing, Optimal local rejection for classifiers, Neurocomputing (2016) 1-13.

[59] W. Arlt, M. Biehl, A. E. Taylor, S. Hahner, R. Libe, B. A. Hughes, P. Schneider, D. J. Smith, H. Stiekema, N. Krone, E. Porfiri, G. Opocher, J. Bertherat, F. Mantero, B. Allolio, M. Terzolo, P. Nightingale, C. H. L. Shackleton, X. Bertagna, M. Fassnacht, P. M. Stewart, Urine steroid 
metabolomics as a biomarker tool for detecting malignancy in adrenal tumors, Journal Clinical Endocrinology and Metabolism 96 (2011) 37753784 .

[60] M. Biehl, P. Schneider, D. Smith, H. Stiekema, A. Taylor, B. Hughes, C. Shackleton, P. Stewart, W. Arlt, Matrix relevance lvq in steroid metabolomics based classification of adrenal tumors., in: ESANN, 2012.

[61] P. Schneider, M. Biehl, B. Hammer, Adaptive relevance matrices in learning vector quantization, Neural Computation 21 (12) (2009) 35323561.

[62] A. Sato, K. Yamada, Generalized learning vector quantization, Advances in neural information processing systems (1996) 423-429.

\section{Appendix}

\subsection{Properties}

\subsubsection{Nonrejected accuracy $A$}

Property II. For the same rejected fraction $r$, we have that if the nonrejected accuracy for $R_{1}$ is greater than the nonrejected accuracy for $R_{2}$, then

$$
A_{R_{1}}(r)=\frac{\left\|\boldsymbol{a}_{\mathcal{N}_{R_{1}}}\right\|}{(1-r) n}>\frac{\left\|\boldsymbol{a}_{\mathcal{N}_{R_{2}}}\right\|}{(1-r) n}=A_{R_{2}}(r)
$$

meaning $R_{1}$ outperforms $R_{2}$.

Property III. If $R_{1}$ outperforms $R_{2}$, for different rejected fractions $r_{1}>r_{2}$, then $\left\|\boldsymbol{a}_{\mathcal{N}_{R_{1}}}\right\|=\left\|\boldsymbol{a}_{\mathcal{N}_{R_{2}}}\right\|$, leading to

$$
A_{R_{1}}\left(r_{1}\right)=\frac{\left\|\boldsymbol{a}_{\mathcal{N}_{R_{1}}}\right\|}{\left(1-r_{1}\right) n}=\frac{\left\|\boldsymbol{a}_{\mathcal{N}_{R_{2}}}\right\|}{\left(1-r_{1}\right) n}>\frac{\left\|\boldsymbol{a}_{\mathcal{N}_{R_{2}}}\right\|}{\left(1-r_{2}\right) n}=A_{R_{2}}\left(r_{2}\right)
$$


If $R_{1}$ outperforms $R_{2}$, for different rejected fractions $r_{1}<r_{2}$, then $\left\|\mathbf{1}-\boldsymbol{a}_{\mathcal{N}_{R_{1}}}\right\|=$ $\left\|\mathbf{1}-\boldsymbol{a}_{\mathcal{N}_{R_{2}}}\right\|$.

$$
\begin{array}{r}
\left\|\mathbf{1}-\boldsymbol{a}_{\mathcal{N}_{R_{1}}}\right\|=\left\|\mathbf{1}-\boldsymbol{a}_{\mathcal{N}_{R_{2}}}\right\| \Longleftrightarrow\left(1-r_{1}\right) n-\left\|\boldsymbol{a}_{\mathcal{N}_{R_{1}}}\right\|=\left(1-r_{2}\right) n-\left\|\boldsymbol{a}_{\mathcal{N}_{R_{2}}}\right\| \Longleftrightarrow \\
\left(1-A_{R_{1}}\left(r_{1}\right)\right)=\frac{\left(1-r_{2}\right)}{\left(1-r_{1}\right)}-\frac{\left(1-r_{2}\right)\left\|\boldsymbol{a}_{\mathcal{N}_{R_{2}}}\right\|}{\left(1-r_{1}\right)\left(1-r_{2}\right)} \Longleftrightarrow\left(1-A_{R_{1}}\left(r_{1}\right)\right)= \\
\frac{\left(1-r_{2}\right)}{\left(1-r_{1}\right)}\left(1-A_{R_{2}}\left(r_{2}\right)\right) \Longleftrightarrow 1-A_{R_{1}}\left(r_{1}\right)<1-A_{r_{2}}\left(r_{2}\right) \Longleftrightarrow A_{R_{1}}\left(r_{1}\right)>A_{R_{2}}\left(r_{2}\right)
\end{array}
$$

Property $I V$. The nonrejected accuracy achieves its maximum, 1 , when $\mathcal{N}=\mathcal{A}$ and $\mathcal{R}=\mathcal{M}$. This maximum is not unique however. Any selection of $\mathcal{N}$ such that $\mathcal{N} \subset \mathcal{A}$ achieves a maximum value of nonrejected accuracy. The minimum of the nonrejected accuracy, 0 , is achieved when $\mathcal{N}=\mathcal{M}$ and $\mathcal{R}=\mathcal{A}$. Any selection of $\mathcal{N}$ such that $\mathcal{N} \subset \mathcal{M}$ achieves a minimum value of nonrejected accuracy.

\subsubsection{Classification quality $Q$}

Property II. With representation of the classification quality in (12), we can note that, for the same rejected fraction $r$ if the classification quality for $R_{1}$ is higher than the classification quality for $R_{2}$, then

$$
\begin{array}{r}
Q_{R_{1}}(r)>Q_{R_{2}}(r) \Longleftrightarrow 2 A_{R_{1}}(r)(1-r)-A(0)>2 A_{R_{2}}(r)(1-r)-A(0) \Longleftrightarrow \\
A_{R_{1}}>A_{R_{1}} \Longleftrightarrow\left\|\boldsymbol{a}_{\mathcal{N}_{R_{1}}}\right\|>\left\|\boldsymbol{a}_{\mathcal{N}_{R_{2}}}\right\|
\end{array}
$$

Property III. If $R_{1}$ outperforms $R_{2}$, for different rejected fractions $r_{1}>r_{2}$, then $\left\|\boldsymbol{a}_{\mathcal{N}_{R_{1}}}\right\|=\left\|\boldsymbol{a}_{\mathcal{N}_{R_{2}}}\right\|$, and

$$
\begin{array}{r}
n Q_{R_{1}}\left(r_{1}\right)=\left\|\boldsymbol{a}_{\mathcal{N}_{R_{1}}}\right\|+\left\|1-\boldsymbol{a}_{\mathcal{R}_{R_{1}}}\right\|=\left\|\boldsymbol{a}_{\mathcal{N}_{R_{1}}}\right\|+\left|\boldsymbol{\mathcal { R }}_{R_{1}}\right|-\left\|\boldsymbol{a}_{\mathcal{R}_{R_{1}}}\right\|= \\
\left\|\boldsymbol{a}_{\mathcal{N}_{R_{1}}}\right\|+r_{1} n-\|\boldsymbol{a}\|+\left|\boldsymbol{a}_{\mathcal{N}_{R_{1}}}\|>\| \boldsymbol{a}_{\mathcal{N}_{R_{1}}}\left\|+r_{2} n-\right\| \boldsymbol{a}\left\|+\mid \boldsymbol{a}_{\mathcal{N}_{R_{1}}}\right\|=\right. \\
\left\|\boldsymbol{a}_{\mathcal{N}_{R_{2}}}\right\|+r_{1} n-\|\boldsymbol{a}\|+\mid \boldsymbol{a}_{\mathcal{N}_{R_{2}}} \|=n Q_{R_{2}}\left(r_{2}\right)
\end{array}
$$


If $R_{1}$ outperforms $R_{2}$, for different rejected fractions $r_{1}<r_{2}$, then $\left\|1-\boldsymbol{a}_{\mathcal{N}_{R_{1}}}\right\|=$ $\left\|1-\boldsymbol{a}_{\mathcal{N}_{R_{2}}}\right\|$, and

$$
\begin{array}{r}
n Q_{R_{1}}\left(r_{1}\right)=\left\|\boldsymbol{a}_{\mathcal{N}_{R_{1}}}\right\|+\left\|1-\boldsymbol{a}_{\mathcal{R}_{R_{1}}}\right\|=\left\|\boldsymbol{a}_{\mathcal{N}_{R_{1}}}\right\|+\left|\boldsymbol{R}_{R_{1}}\right|-\left\|\boldsymbol{a}_{\mathcal{R}_{R_{1}}}\right\|= \\
\left|\mathcal{N}_{R_{1}}\right|+\left|\boldsymbol{\mathcal { R }}_{R_{1}}\right|-\left\|1-\boldsymbol{a}_{\mathcal{N}_{R_{1}}}\right\|-\left(\|\boldsymbol{a}\|-\left\|\boldsymbol{a}_{\mathcal{N}_{R_{1}}}\right\|\right)= \\
\left|\mathcal{N}_{R_{1}}\right|+\left|\boldsymbol{\mathcal { R }}_{R_{1}}\right|-\left\|1-\boldsymbol{a}_{\mathcal{N}_{R_{1}}}\right\|-\|\boldsymbol{a}\|+\left|\mathcal{N}_{R_{1}}\right|-\left\|1-\boldsymbol{a}_{\mathcal{N}_{R_{1}}}\right\|= \\
n-A(0)+\left|\mathcal{N}_{R_{1}}\right|-2\left\|1-\boldsymbol{a}_{\mathcal{N}_{R_{1}}}\right\|>n-A(0)+\left|\mathcal{N}_{R_{2}}\right|-2\left\|1-\boldsymbol{a}_{\mathcal{N}_{R_{1}}}\right\|= \\
n-A(0)+\left|\mathcal{N}_{R_{2}}\right|-2\left\|1-\boldsymbol{a}_{\mathcal{N}_{R_{2}}}\right\|=n Q_{R_{2}}\left(r_{2}\right)
\end{array}
$$

Property $I V$. The classification quality achieves its unique maximum, 1 , if $\mathcal{A}=\mathcal{N}$ and $\mathcal{M}=\mathcal{R}$. Conversely, it achieves its unique minimum, 0 , if $\mathcal{A}=\mathcal{R}$ and $\mathcal{M}=\mathcal{N}$.

\subsubsection{Rejection quality $\phi$}

Property II. For the same rejected fraction $r$, we have that if the rejection quality for $R_{1}$ is greater than the rejection quality for $R_{2}$, then

$$
\begin{aligned}
& \phi_{R_{1}}(r)>\phi_{R_{2}}(r) \Longleftrightarrow \frac{\left\|1-\boldsymbol{a}_{\mathcal{R}_{R_{1}}}\right\|}{\left\|\boldsymbol{a}_{\mathcal{R}_{R_{1}}}\right\|} \frac{\|\boldsymbol{a}\|}{\|1-\boldsymbol{a}\|}>\frac{\left\|1-\boldsymbol{a}_{\mathcal{R}_{R_{2}}}\right\|}{\left\|\boldsymbol{a}_{\mathcal{R}_{R_{2}}}\right\|} \frac{\|\boldsymbol{a}\|}{\|1-\boldsymbol{a}\|} \Longleftrightarrow \\
& \frac{\left\|1-\boldsymbol{a}_{\mathcal{R}_{R_{1}}}\right\|}{\left\|\boldsymbol{a}_{\mathcal{R}_{R_{1}}}\right\|}>\frac{\left\|1-\boldsymbol{a}_{\mathcal{R}_{R_{2}}}\right\|}{\left\|\boldsymbol{a}_{\mathcal{R}_{R_{2}}}\right\|} \frac{\left|\mathcal{R}_{R_{1}}\right|-\left\|\boldsymbol{a}_{\mathcal{R}_{R_{1}}}\right\|}{\left\|\boldsymbol{a}_{\mathcal{R}_{R_{1}}}\right\|}>\frac{\left|\mathcal{R}_{R_{2}}\right|-\left\|\boldsymbol{a}_{\mathcal{R}_{R_{2}}}\right\|}{\left\|\boldsymbol{a}_{\mathcal{R}_{R_{2}}}\right\|} \Longleftrightarrow \\
& \frac{\left|\mathcal{R}_{R_{1}}\right|-\left\|\boldsymbol{a}_{\mathcal{R}_{R_{1}}}\right\|}{\left\|\boldsymbol{a}_{\mathcal{R}_{R_{1}}}\right\|}>\frac{\left|\mathcal{R}_{R_{2}}\right|-\left\|\boldsymbol{a}_{\mathcal{R}_{R_{2}}}\right\|}{\left\|\boldsymbol{a}_{\mathcal{R}_{R_{2}}}\right\|} \frac{\left|\mathcal{R}_{R_{1}}\right|}{\left\|\boldsymbol{a}_{\mathcal{R}_{R_{1}}}\right\|}-1>\frac{\left|\mathcal{R}_{R_{2}}\right|}{\left\|\boldsymbol{a}_{\mathcal{R}_{R_{2}}}\right\|}-1 \Longleftrightarrow \\
& \frac{\left\|\boldsymbol{a}_{\mathcal{R}_{R_{1}}}\right\|}{\left|\mathcal{R}_{R_{1}}\right|}<\frac{\left\|\boldsymbol{a}_{\mathcal{R}_{R_{2}}}\right\|}{\left|\mathcal{R}_{R_{1}}\right|}\|\boldsymbol{a}\|-\left\|\boldsymbol{a}_{\mathcal{N}_{R_{1}}}\right\|<\|\boldsymbol{a}\|-\left\|\boldsymbol{a}_{\mathcal{N}_{R_{2}}}\right\| \Longleftrightarrow\left\|\boldsymbol{a}_{\mathcal{N}_{R_{1}}}\right\|>\left\|\boldsymbol{a}_{\mathcal{N}_{R_{2}}}\right\|
\end{aligned}
$$

Property III. If $R_{1}$ outperforms $R_{2}$, for different rejected fractions $r_{1}>r_{2}$, then $\left\|\boldsymbol{a}_{\mathcal{N}_{R_{1}}}\right\|=\left\|\boldsymbol{a}_{\mathcal{N}_{R_{2}}}\right\|$. As $\|\boldsymbol{a}\|=\left\|\boldsymbol{a}_{\mathcal{N}}\right\|+\left\|\boldsymbol{a}_{\mathcal{R}}\right\|$ and $r_{1}>r_{2}$, we have $\left\|\boldsymbol{a}_{\mathcal{R}_{R_{1}}}\right\|=$ $\| \boldsymbol{a}_{\mathcal{R}_{R_{2}}}||$ and $\left|\mathcal{R}_{R_{1}}\right|>\left|\mathcal{R}_{R_{2}}\right|$ respectively, leading to

$$
\begin{gathered}
\phi_{R_{1}}\left(r_{1}\right)=\frac{\left\|1-\boldsymbol{a}_{\mathcal{R}_{R_{1}}}\right\|}{\left\|\boldsymbol{a}_{\mathcal{R}_{R_{1}}}\right\|} \frac{\|\boldsymbol{a}\|}{\|1-\boldsymbol{a}\|}=\left(\frac{\left|\mathcal{R}_{R_{1}}\right|}{\left\|\boldsymbol{a}_{\mathcal{R}_{R_{1}}}\right\|}-1\right) \frac{\|\boldsymbol{a}\|}{\|1-\boldsymbol{a}\|}> \\
>\left(\frac{\left|\mathcal{R}_{R_{2}}\right|}{\left\|\boldsymbol{a}_{\mathcal{R}_{R_{1}}}\right\|}-1\right) \frac{\|\boldsymbol{a}\|}{\|1-\boldsymbol{a}\|}=\frac{\left\|1-\boldsymbol{a}_{\mathcal{R}_{R_{2}}}\right\|}{\left\|\boldsymbol{a}_{\mathcal{R}_{R_{2}}}\right\|} \frac{\|\boldsymbol{a}\|}{\|1-\boldsymbol{a}\|}=\phi_{R_{2}}\left(r_{2}\right)
\end{gathered}
$$


If $R_{1}$ outperforms $R_{2}$, for different rejected fractions $r_{1}<r_{2}$, i.e. $\left|\mathcal{N}_{R_{1}}\right|>\left|\mathcal{N}_{R_{2}}\right|$, then $\left\|1-\boldsymbol{a}_{\mathcal{N}_{R_{1}}}\right\|=\left\|1-\boldsymbol{a}_{\mathcal{N}_{R_{2}}}\right\|$. This means that $\left\|1-\boldsymbol{a}_{\mathcal{R}_{R_{1}}}\right\|=\left\|1-\boldsymbol{a}_{\mathcal{R}_{R_{2}}}\right\|$ and $\left|\mathcal{R}_{1}\right|<\left|\mathcal{R}_{2}\right|$

$$
\begin{array}{r}
\phi_{R_{1}}\left(r_{1}\right)=\frac{\left\|1-\boldsymbol{a}_{\mathcal{R}_{R_{1}}}\right\|}{\left\|\boldsymbol{a}_{\mathcal{R}_{R_{1}}}\right\|} \frac{\|\boldsymbol{a}\|}{\|1-\boldsymbol{a}\|}=\frac{\left\|1-\boldsymbol{a}_{\mathcal{R}_{R_{1}}}\right\|}{\left|\mathcal{R}_{R_{1}}\right|-\left\|1-\boldsymbol{a}_{\mathcal{R}_{R_{1}}}\right\|} \frac{\|\boldsymbol{a}\|}{\|1-\boldsymbol{a}\|}> \\
\frac{\left\|1-\boldsymbol{a}_{\mathcal{R}_{R_{1}}}\right\|}{\left|\mathcal{R}_{R_{2}}\right|-\left\|1-\boldsymbol{a}_{\mathcal{R}_{R_{1}}}\right\|} \frac{\|\boldsymbol{a}\|}{\|1-\boldsymbol{a}\|}=\frac{\left\|1-\boldsymbol{a}_{\mathcal{R}_{R_{2}}}\right\|}{\left|\mathcal{R}_{R_{2}}\right|-\left\|1-\boldsymbol{a}_{\mathcal{R}_{R_{2}}}\right\|} \frac{\|\boldsymbol{a}\|}{\|1-\boldsymbol{a}\|}=\phi_{R_{2}}\left(r_{2}\right) \quad \square
\end{array}
$$

Property $I V$. The rejection quality achieves its maximum, $\infty$, when $\mathcal{N}=\mathcal{A}$ and $\mathcal{R}=\mathcal{M}$. This maximum is not unique. Any selection of $\mathcal{R}$ such that $\mathcal{R} \subset \mathcal{M}$ results in maximum values of rejection quality. Conversely, the rejection quality achieves its minimum, 0 , when $\mathcal{R}=\mathcal{A}$ and $\mathcal{N}=\mathcal{M}$. This maximum is not unique. Any selection of $\mathcal{R}$ such that $\mathcal{R} \subset \mathcal{A}$ results in minimum values of rejection quality.

\subsection{Comparing performance of classifiers with rejection}

Let us consider a classifier $C$ and two rejectors $R_{1}$ and $R_{0}$, with $r_{1}>r_{0}$, and a cost function with a rejection-misclassification trade-off $\rho$. Let $\beta$ be the relative optimality of the operating point of rejector $R_{1}$ at $r_{1}$ with respect to the reference operating point of $R_{0}$ at $r_{0}$.

From (22), and given the cost function with a rejection-misclassification tradeoff $\rho$, we can relate outperformance, $\beta$ and $\rho$ : rejector $R_{1}$ outperforms $R_{0}$ when $\beta>2 \rho-1$; rejector $R_{0}$ outperforms $R_{1}$ when $\beta<2 \rho-1$; rejector $R_{0}$ and $R_{1}$ are equivalent in terms of performance when $\beta=2 \rho-1$.

\subsubsection{Nonrejected accuracy $A$}

We can represent $A_{R_{1}}\left(r_{1}\right)$ as a function of $A_{R_{0}}\left(r_{0}\right)$ by noting that the best case scenario is $A_{R_{1}}\left(r_{1}\right)=A_{R_{0}}\left(r_{0}\right) \frac{1-r_{0}}{1-r_{1}}+\frac{r_{1}-r_{0}}{1-r_{1}}$, corresponding to $\beta=1$, and the worst case scenario is $A_{R_{1}}\left(r_{1}\right)=A_{R_{0}}\left(r_{0}\right) \frac{1-r_{0}}{1-r_{1}}$, corresponding to $\beta=-1$. 
This results in a representation of the nonrejected accuracy $A_{R_{1}}\left(r_{1}\right)$ as $A_{R_{1}}\left(r_{1}\right)=$ $A_{R_{0}}\left(r_{0}\right) \frac{1-r_{0}}{1-r_{1}}+\frac{\beta-1}{2} \frac{r_{1}-r_{0}}{1-r_{1}}$.

Rejector $R_{1}$ outperforms $R_{0}$ when

$$
A_{R_{1}}\left(r_{1}\right)=A_{R_{0}}\left(r_{0}\right) \frac{1-r_{0}}{1-r_{1}}+\frac{\beta-1}{2} \frac{r_{1}-r_{0}}{1-r_{1}}>A_{R_{0}}\left(r_{0}\right) \frac{1-r_{0}}{1-r_{1}}+(\rho-1) \frac{r_{1}-r_{0}}{1-r_{1}} .
$$

Rejector $R_{0}$ outperforms $R_{1}$ when

$$
A_{R_{1}}\left(r_{1}\right)=A_{R_{0}}\left(r_{0}\right) \frac{1-r_{0}}{1-r_{1}}+\frac{\beta-1}{2} \frac{r_{1}-r_{0}}{1-r_{1}}<A_{R_{0}}\left(r_{0}\right) \frac{1-r_{0}}{1-r_{1}}+(\rho-1) \frac{r_{1}-r_{0}}{1-r_{1}} .
$$

Rejector $R_{0}$ and $R_{1}$ are equivalent in terms of performance when

$$
A_{R_{1}}\left(r_{1}\right)=A_{R_{0}}\left(r_{0}\right) \frac{1-r_{0}}{1-r_{1}}+\frac{\beta-1}{2} \frac{r_{1}-r_{0}}{1-r_{1}}=A_{R_{0}}\left(r_{0}\right) \frac{1-r_{0}}{1-r_{1}}+(\rho-1) \frac{r_{1}-r_{0}}{1-r_{1}} .
$$

\subsubsection{Classification quality $Q$}

We can represent $Q_{R_{1}}\left(r_{1}\right)$ as a function of $Q_{R_{0}}\left(r_{0}\right)$ by noting that the best case scenario is $Q_{R_{1}}\left(r_{1}\right)=Q_{R_{0}}\left(r_{0}\right)+\left(r_{1}-r_{0}\right)$, corresponding to $\beta=1$, and the worst case scenario is $Q_{R_{1}}\left(r_{1}\right)=Q_{R_{0}}\left(r_{0}\right)-\left(r_{1}-r_{0}\right)$, corresponding to $\beta=-1$. This results in a representation of the classification quality $Q\left(R_{1}\right)\left(r_{1}\right)$ as $Q_{R_{1}}\left(r_{1}\right)=$ $Q_{R_{0}}\left(r_{0}\right)+\beta\left(r_{1}-r_{0}\right)$.

Rejector $R_{1}$ outperforms $R_{0}$ when $Q_{R_{1}}\left(r_{1}\right)=Q_{R_{0}}\left(r_{0}\right)+\beta\left(r_{1}-r_{0}\right)>Q_{R_{0}}\left(r_{0}\right)+$ $(2 \rho-1)\left(r_{1}-r_{0}\right)$.

Rejector $R_{0}$ outperforms $R_{1}$ when $Q_{R_{1}}\left(r_{1}\right)=Q_{R_{0}}\left(r_{0}\right)+\beta\left(r_{1}-r_{0}\right)<Q_{R_{0}}\left(r_{0}\right)+$ $(2 \rho-1)\left(r_{1}-r_{0}\right)$.

Rejector $R_{0}$ and $R_{1}$ are equivalent in terms of performance when $Q_{R_{1}}\left(r_{1}\right)=$ $Q_{R_{0}}\left(r_{0}\right)+\beta\left(r_{1}-r_{0}\right)=Q_{R_{0}}\left(r_{0}\right)+(2 \rho-1)\left(r_{1}-r_{0}\right)$. 
performance measures for

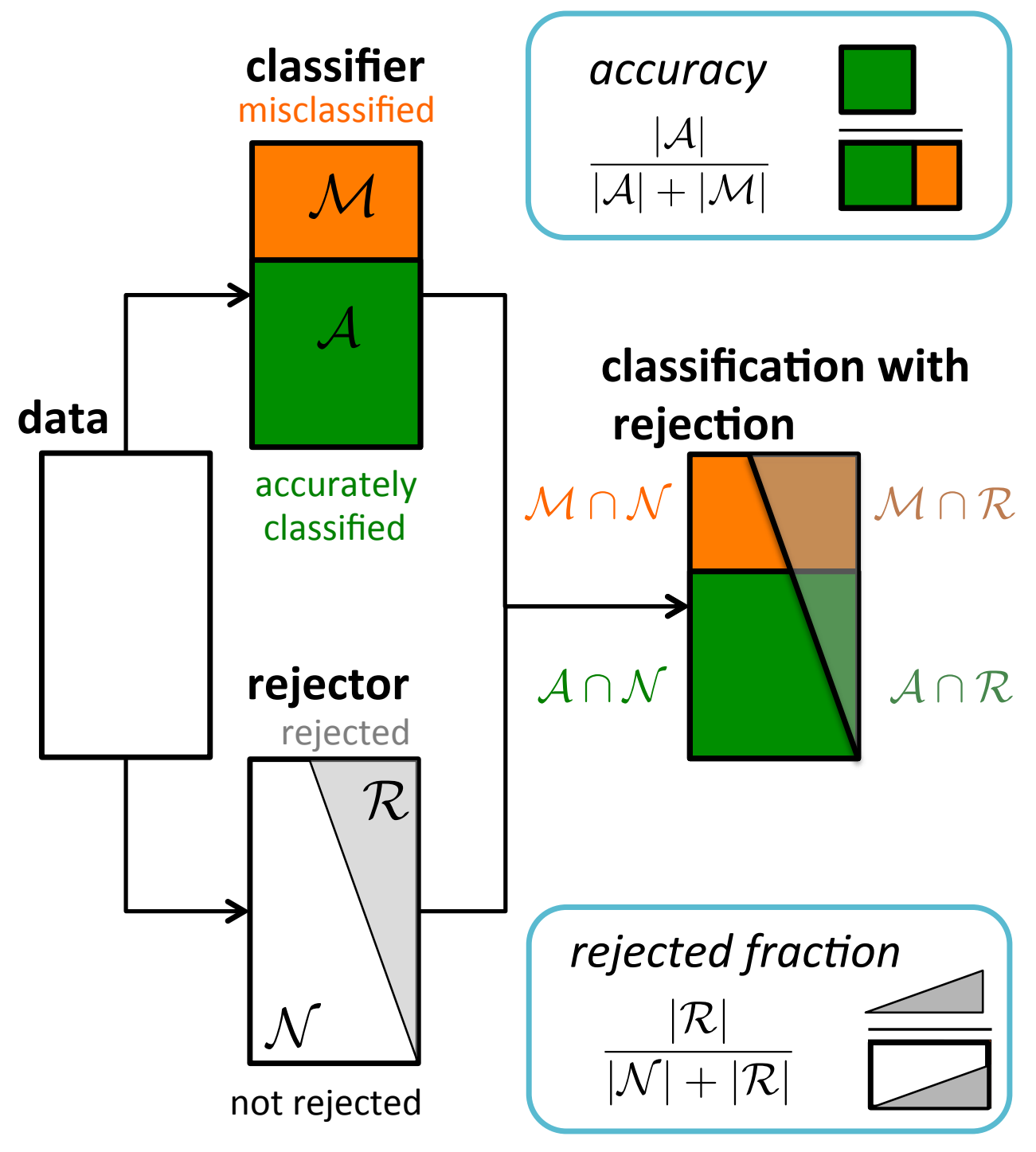
classification systems with rejection

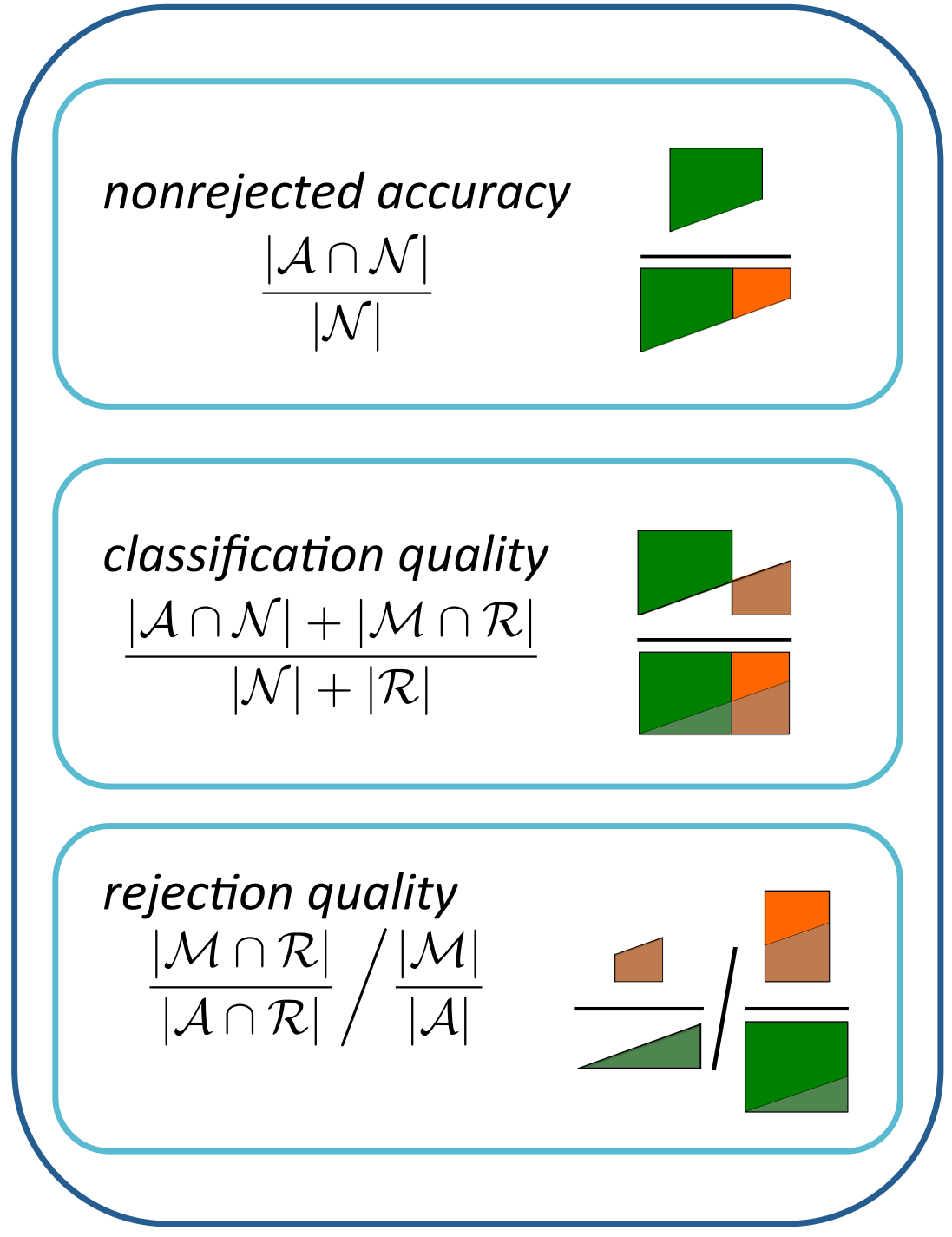

